\title{
De l'harmonie dans la construction des mots français
}

\author{
Michel Roché ${ }^{1}$ et Marc Plénat ${ }^{2}$ \\ ${ }^{1}$ Université Toulouse Jean Jaurès \\ ${ }^{2} \mathrm{CLVA}$ \\ mroche@univ-tlse2.fr, plenat@univ-tlse2.fr
}

\begin{abstract}
Résumé. La formation des gentilés, particulièrement sensible aux contraintes d'euphonie, permet de mettre en évidence, pour le français, deux manifestations complémentaires de la contrainte dissimilative. L'une, négative, tend à éviter la consécution à faible distance du même phonème ou de deux phonèmes proches. Elle explique la sous-représentation des deux principaux suffixes, -ais et -ois, après une accroche en sifflante, à cause de la sifflante contenue par le suffixe au féminin, ainsi que la sous-représentation de -ais après /E/ et de -ois après /A/. L'autre, positive, favorise entre les voyelles une harmonie fondée sur les contrastes. Devant les suffixes dont la voyelle est une voyelle d'avant moyenne ou haute (-ais, -ain, -in, -ier...), sont privilégiés les radicaux dont la dernière voyelle est une voyelle d'arrière moyenne ou basse, et de préférence un /O/ plutôt qu'un /A/. Devant les suffixes dont la voyelle est une voyelles d'arrière moyenne ou basse (-ois, -ot, -on, -and, -at, -ard...), sont privilégiés les radicaux dont la dernière voyelle est une voyelle d'avant moyenne ou haute, et de préférence un $/ \mathrm{i}$ / plutôt qu'un /E/. Pour cela, un yod au contact du suffixe joue le même rôle qu'un /i/ dans la syllabe précédente. Cette harmonie est obtenue soit par la sélection du suffixe, soit par un aménagement - substitution, épenthèse, troncation, insertion d'un interfixe, etc. - du radical fourni par la base. On observe d'autre part une harmonisation de l'accroche consonantique du radical avec la voyelle du suffixe. Devant -ois, sont privilégiées les attaques en labiale ou en vélaire, en accord avec le double caractère du /w/ propre à ce suffixe. Devant -ais et -ain, ce sont les attaques en dentale. Devant -ot, -on, -and, -at, -ard, la tendance à rapprocher l'articulation de la consonne de celle de la voyelle peut aller jusqu'aux vélaires mais elle s'arrête souvent aux palatales à cause de la rareté des moyens offerts par les vélaires en français et de la contrainte portant sur les voyelles, qui privilégie devant ces suffixes une attaque en yod.
\end{abstract}

\begin{abstract}
The formation of French gentilics, which is particularly sensitive to euphonic constraints, displays two complementary manifestations of the dissimilative constraint. One is negative, and tends to avoid the immediate repetition of two identical or similar phonemes. It explains, for example: (i) the underrepresentation of the two most common suffixes -ais and -ois after a sibilant, due to the presence of the sibilant in the feminine suffix; (ii) the underrepresentation of -ais after /E/ and of -ois after /A/. The other is positive and favours vowel harmony based on their contrasts. Before suffixes whose vowel is a high or mid front vowel (-ais, -ain, -in, -ier...), one is more likely to find stems whose final vowel is a low or mid back vowel, with a preference for $/ \mathrm{O} /$ rather than /A/, while suffixes whose vowel is a low or mid back vowel (-ois, -ot, -on, -and, -at, -ard...) are more likely to be preceded by stems whose vowel is a high or mid front vowel, with a preference for /i/ rather than /E/. A yod before the suffix plays the same role as an /i/ in the preceding syllable. This harmony is achieved either by the choice of suffix, or by an adjustment of the stem furnished by the base through segmental substitution, epenthesis, truncation, insertion of an interfix, etc. It is also noticeable that the onset provided by the radical is harmonized with the vowel of the suffix. A labial or
\end{abstract}


velar onset is preferred before -ois, in accordance with the two place features of the /w/ peculiar to this suffix, whereas a dental onset is preferred before -ais and -ain. Although the tendency to assimilate the articulation of the consonant to that of the vowel before -ot, -on, -and, -at, -ard may go back as far as the velars, it often stops with the palatals, due not only to the extremely limited resources offered by the velars in French, but also to the constraint bearing on the vowels, which favours yod insertion before these suffixes.

On se propose de montrer qu'il existe vraisemblablement en français une contrainte de «bonne formation » qui tend à opposer, dans les deux dernières syllabes d'un mot construit, une voyelle haute et/ou d'avant à une voyelle basse et/ou d'arrière. Peu apparente, en général, dans la mesure où elle est contrariée par d'autres contraintes plus fortes qu'elle, on peut néanmoins la mettre en évidence dans la formation des gentilés. Ils offrent pour cela plusieurs avantages. Ils sont très nombreux (on a pu rassembler dans une base de données plus de 40000 formes), et le nombre lui-même rend visibles des faits peu perceptibles à plus petite échelle, ou permet de confirmer de simples intuitions. Ils sont formés au moyen d'un grand nombre de suffixes ${ }^{1}$, spécialisés (-ois, -ais, -ien, -ain, -éen) ou non (-in, -iste, -ot, -on, -at, -ard, -and, etc.), tous strictement équivalents, dans ce rôle, sur le plan sémantique. De ce fait, le choix de l'un ou de l'autre est libre pour satisfaire - inconsciemment - d'autres contraintes. Occupant, enfin, une place marginale dans la création lexicale, ils échappent plus facilement à la vigilance des gardiens de la norme et aux contraintes de régularité. En plus du choix du suffixe, celui de la forme donnée au radical est donc très libre et l'on peut y observer toutes sortes d'aménagements morphophonologiques.

De nombreux travaux ont été consacrés aux gentilés mais peu, à l’exception de Plénat (2008), les abordent sous l'angle privilégié ici. On renverra à cet article et à Eggert (2002) pour une revue de la littérature antérieure. On esquissera d'abord succinctement les caractéristiques générales de la formation des gentilés en français pour situer la question de l'harmonie par rapport aux autres paramètres qui entrent en jeu. Le cadre d'analyse sera celui d'une morphologie dérivationnelle fondée sur la notion de contrainte plutôt que sur celle de Règle de Construction des Mots. On s'intéressera peu aux dérivations en -ien et en -éen qui, à cause de leur caractère "savant ", échappent en grande partie aux contraintes phonologiques qu'il s'agit d'étudier. Parmi les formes populaires, l'approche que nous avons choisie permettra de renouveler la question souvent débattue de la distribution des deux principales, -ais et -ois. L'accès à un plus vaste corpus permettra surtout d'observer ce qui se passe pour les gentilés formés avec d'autres suffixes (-ot, -on, -at, -ard en particulier), qu'on mentionnait sans pouvoir les étudier parce qu'on ne disposait pas d'un nombre de formes assez important. On verra qu'ils éclairent utilement l'ensemble du tableau.

\section{$1 \quad$ La formation des gentilés}

Par convention, on appellera gentilés les noms d’humains qui dénotent l'appartenance des référents à une entité géographique (ville, village, pays, province...) et qui sont construits, normalement, à partir du nom de cette entité : Lyon $\rightarrow$ Lyonnais, Norvège $\rightarrow$ Norvégien. Et ethniques les noms d'humains qui dénotent l'appartenance des référents à un peuple, une ethnie, etc., indépendamment d'un ancrage territorial, qui est le plus souvent absent (Touareg, Apache) et second quand il existe (Turc $\rightarrow$ Turquie, Kazakh $\rightarrow \mathrm{Ka}$ zakhstan). On ne s’occupera ici que des gentilés définis comme ci-dessus.

Comme les autres formations, celle des gentilés obéit à un certain nombre de contraintes et dépend du cahier des charges - généralement implicite - qui a présidé à leur création. Pour les contraintes, on utilisera le cadre descriptif de Roché et Plénat (2014), et pour le cahier des charges on distinguera trois cas de figure, suivant qu'il s'agit de former un gentilé standard, un gentilé plus distingué ou bien un gentilé ludique ou idiosyncrasique.

Pour satisfaire la contrainte de série, un gentilé standard devra être terminé par -ois ou par -ais puisque les séries dérivationnelles qui rassemblent la grande majorité des gentilés de ce type sont caractérisées par ces deux finales. Ce résultat est obtenu le plus souvent par suffixation (1a). Mais si le toponyme est terminé lui-même par -ois ou par -ais la contrainte de série sera satisfaite si le gentilé garde la même forme, moyennant une alternance thématique pour former le féminin (1b). Ce procédé peut s'accommoder d’un 
écart graphique (1c) ou entraîner des troncations internes (1d) et même un remaniement du matériau phonologique (1e).

(1a) GOURIN (56) $\rightarrow$ Gourinois, -oise

(1b) MONTENOIS (25) $\rightarrow$ Montenois, -oise

(1c) SAINT-LIEFFROY (25) $\rightarrow$ Lieffrois, -oise

(1d) SAINT-PIERRE-DU-PALAIS (17) $\rightarrow$ Pierre

(1e) LiMOILOU (Q) $\rightarrow$ Limoulois, -oise

Si le toponyme a une finale suffixale ou suffixoïde qui suit une accroche pouvant devenir la finale d'un gentilé, celui-ci pourra être obtenu par dérivation régressive (2).

(2) Tournolsis (45) $\rightarrow$ Tournois -oise

Pour former un gentilé plus distingué, la contrainte de série prescrira une dérivation en -ien, non pour le suffixe lui-même mais parce que les dérivés en -ien présents dans le lexique sont construits en majorité sur un thème savant. Comme l'essentiel est de "faire savant ", le radical latin pourra être bricolé et les règles de la morphologie classique quelque peu bousculées (3).

(3) CHAMPDOLENT (17) $\rightarrow$ Campodoloricien, -ienne LA CROIX-EN-TOURAINE (37) $\rightarrow$ Crucifixien, -ienne VIEILLES-MAISONS (45) $\rightarrow$ Vetula-domussien, -ienne

Pour former un gentilé ludique, tout est permis et le morphologue ne peut que constater le résultat. Certains sont plutôt des surnoms, sans relation avec le toponyme, donc hors série (4).

(4) SAINT-JEAN-AUX-Bois (60) / Solitaires COSSONAY (Sr) / Traîne-Gourdins

Sans intention ludique, d'autres peuvent entrer dans l'une ou l'autre des grandes séries dérivationnelles mais sont également inanalysables parce que construits sur une dénomination ancienne de la localité (5a) ou sur d’autres types de bases supplétives (5b).

(5a) DiEUlOUARD (54) / Scarponnais, -aise

(5b) Meisenthal (57) / Val-Mésangeois, -oise [cf. al. Meise 'mésange’, Tal 'vallée’]

En plus des trois grandes séries et des gentilés atypiques, l’héritage latin et les emprunts divers ont laissé de nombreuses traces, amorces de séries moins étendues caractérisées par d'autres suffixes. Séries qui peuvent elles-mêmes attirer des formations nouvelles et remplacer -ois, -ais ou -ien quand d'autres contraintes les rendent indésirables. Y compris en gardant la même forme (6a), sauf alternance thématique ${ }^{2}$, ou par dérivation régressive (6b), et des gentilés ludiques qui jouent sur l'homonymie du toponyme et/ou du dérivé avec un nom commun (6c).

(6a) FILAIN (70) $\rightarrow$ Filain, -aine VALLORCINE (74) $\rightarrow$ Vallorcin, -ine VELLEROT (25) $\rightarrow$ Vellerot, -ote

(6b) LA GUYONNIERE (85) $\rightarrow$ Guyon, -onne (6c) LURE (70) $\rightarrow$ Luron, -onne AuMONT (39) $\rightarrow$ Aumonier, -ière GRACES (22) $\rightarrow$ Gracieux, -euse LA LOUPE (28) $\rightarrow$ Loupiot, -ote

A l'intérieur de ces grandes séries, la même logique lexicale aboutit à la constitution de sous-séries dans lesquelles un voisinage géographique ou une identité phonologique déterminent le choix du suffixe ou la forme du radical. C'est une expansion en tache d'huile qui explique en grande partie la répartition des deux variantes du même suffixe que sont, historiquement, -ois et -ais. On a remarqué depuis longtemps que cette répartition est très inégale suivant les régions et cherché à la corréler avec les évolutions dialectales de l'ancienne diphtongue /wE/ en direction de -ais ou de -ois. Sans résultats probants. Quelle que soit la raison qui a fait pencher tel ou tel gentilé ancien d'un côté ou de l'autre, il en a vraisemblablement entraîné d'autres plus récents autour de lui ${ }^{3}$. La prédominance de -ois en Alsace, où il est hégémonique, est peut-être due simplement au modèle fourni par Strasbourgeois, la plupart des autres localités n’ayant pas d'autre gentilé, jusqu'à il y a peu, que celui en -er du dialecte germanique ${ }^{4}$. Or Strasbourgeois est dicté lui-même par le modèle de bourg $\rightarrow$ bourgeois. 
On sait (cf. Roché et Plénat 2014, entre autres) qu'en matière de morphologie suffixale une rime riche vaut mieux qu'une rime pauvre ou suffisante. Une sous-série dérivationnelle dans laquelle l'homophonie porte non seulement sur le suffixe mais aussi sur la syllabe qui précède attire d'autant plus les nouveaux entrants. Et plus encore quand y figure un leader word de grande diffusion. C'est ainsi que la plupart des nombreux toponymes en -cour(t) donnent des dérivés en -courtois, comme ceux en -bourg des dérivés en -bourgeois. La finale -itain, qui suit -pol- dans un certain nombre de gentilés exotiques (Napolitain, Constantinopolitain), s'est diffusée ainsi dans des dénominations pseudo-savantes où -pol(is) remplace -ville (ChARLEVILle (08) $\rightarrow$ Carolopolitain) puis pour des toponymes où cette séquence n'est due qu'à une homophonie (SAINT-Pol-DE-LEON (29) $\rightarrow$ Saint-Politain, MESPAUl (29) $\rightarrow$ Mespaulitain). Et puisque les habitants de NAPLES sont les Napolitains, ceux de CANAPLES (80) seront les Canapolitains. La finale -icain, familière dans Américain où elle suit -ér-, passera pour cette raison dans Valéricain $(\leftarrow$ SAINTVALERY-SUR-SOMME (80)) et la finale -isien de Parisien dans Barisien ( $\leftarrow$ BAR-LE-DUC (55)). Etc., etc.

La contrainte de famille - qui veut que soit choisi, comme radical d'un nouveau dérivé, un thème déjà présent dans la famille dérivationnelle du lexème base - est rarement activée dans la formation des gentilés puisque, le plus souvent, le toponyme n'a pas servi de base à d'autres dérivations. Mais s'il a luimême été formé à partir d'un lexème qui reste reconnaissable, celui-ci pourra lui transmettre son thème présuffixal. Ainsi CHAUfFour (19) hérite-t-il de l'alternance /ur/ /urn/ de Four $\rightarrow$ fourneau, chaufournier pour donner Chauffournais, malgré la graphie. En l'absence de co-dérivés antérieurs, ce sont les contraintes de fidélité à la forme libre qui entrent en jeu - fidélité à la forme sonore ou, plus souvent, à la forme écrite. Les habitudes graphiques du français faisant que les consonnes latentes - absentes de la forme libre mais présentes dans le thème présuffixal populaire - soient inscrites dans la graphie, une consonne finale graphique, muette dans le toponyme lui-même, sera interprétée comme une consonne latente et mobilisée dans le radical du gentilé. Ainsi le - $d$ final de DiNARD (qui n’est pas étymologique) sera-t-il actualisé dans Dinardais. La fidélité à la forme libre entre souvent en conflit avec la contrainte de famille. Les toponymes en -vaux, par exemple, donnent tantôt des gentilés en -val- qui activent le même thème que vallée, vallon (GRANDVAUX (71) $\rightarrow$ Grandvallois), tantôt des gentilés en -vaut- ou en -vaud-, plus proches de la forme orale, avec une consonne épenthétique (VAUX (03) $\rightarrow$ Vaudois), tantôt des gentilés en -vaux- qui conservent la forme écrite alors que le $-x$, d’origine flexionnelle, n'entre pas normalement dans l'alternance thématique (VAUX-SUR-SEINE (78) $\rightarrow$ Vauxois).

La contrainte de registre veut que -ais et -ois, comme les autres suffixes populaires, sélectionnent le thème présuffixal populaire. Elle est le plus souvent respectée, sauf quand le thème savant permet de satisfaire d'autres contraintes (infra 3.1), ou bien quand, malgré un désir de distinction, d'autres contraintes s'opposent à une dérivation en -ien (après une accroche en /n/, par exemple). Symétriquement, -ien ou -éen seront précédés d'un thème populaire lorsqu'ils sont choisis non pour eux-mêmes mais parce que des contraintes phonologiques rendaient -ais ou -ois indésirables.

Parmi les contraintes phonologiques, on mentionnera simplement pour mémoire la contrainte de syllabation et la contrainte de taille. La première conduit à fournir une accroche consonantique, par épenthèse ou troncation, aux thèmes présuffixaux qui en sont dépourvus (GUEHENNO (56) $\rightarrow$ Guéhennotais, RicHELIEU (37) $\rightarrow$ Richelais). La seconde, qui privilégie, dans la suffixation populaire, les radicaux dissyllabiques et les dérivés trisyllabiques, conduit à l'insertion d'un interfixe qui allonge le radical (GLAND (89) $\rightarrow$ Glandinois) ou au choix du suffixe dissyllabique -éen (TrEBES (11) $\rightarrow$ Trébéen). Les autres contraintes phonologiques font l’objet de la présente communication.

\section{Corpus et méthode}

La base de données sur laquelle repose ce travail a été constituée à partir de diverses sources, principalement les listes qui figurent dans les grands dictionnaires ou qu'on peut trouver sur la Toile. Les relevés anciens de Merlet (1882) et de Rolland de Denus (1889) ont permis d'y faire entrer un certain nombre de dénominations populaires remplacées depuis par des gentilés plus tendance.

Pour plus de cohérence, on a distingué deux corpus : (1) les gentilés associés à des toponymes de la France métropolitaine (sauf Corse, Alsace et Moselle) et des DOM-TOM, plus ceux de la Belgique wallonne, de la Suisse romande, du Val d'Aoste, des îles Anglo-Normandes et du Québec ${ }^{5}$; (2) les gentilés associés aux toponymes d'autres pays ou régions. Dans le corpus 2, de nombreux gentilés sont des adap- 
tations de formes étrangères, ou construits selon des modalités morphophonologiques différentes de celles du français, et de ce fait risquent de fausser les observations d'ensemble. En toute rigueur, plutôt que ce découpage géographique, on aurait dû distinguer d'un côté les gentilés construits sur des toponymes dont la forme est française ou francisée et selon des modalités morphophonologiques proprement françaises (il y en a dans le corpus 2), de l'autre les toponymes dont la forme reste étrangère et les gentilés construits selon d'autres modalités (il y en a dans le corpus 1). Mais la frontière entre ces deux ensembles est loin d'être nette et la distinction aurait été souvent difficile à établir. Compte tenu de l'ampleur des données, il ne semble pas que les quelques formes suspectes du corpus 1 entachent les observations d'ensemble.

Chacun des deux ensembles a été subdivisé en deux : les corpus 1.1 et 2.1 ne contiennent que les gentilés construits par suffixation sur le toponyme ; les corpus 1.2 et 2.2 rassemblent tous les autres, en particulier les gentilés sans relation morphologique avec le toponyme. Ont été éliminées les variantes seulement graphiques ou sans intérêt morphophonologique. Toutes les observations qui suivent ont été faites sur le corpus 1.1, qui contient 34790 formes.

Pour l'analyse des données, on s'appuiera sur la distinction entre thème et radical (Roché, 2010) et sur la notion d'espace thématique introduite par Bonami et Boyé (2003, entre autres) pour la flexion verbale et adaptée depuis à la dérivation dans divers travaux. Pour chaque nom, est inscrit dans le lexique, en plus de la forme libre (thème $A$ ), un thème présuffixal (thème $B$ ) manifesté dans ses dérivés populaires. Ainsi pour POT /po/ le dérivé potier sera construit sur le thème $B$ /pot/. Le thème $B$ est susceptible de prendre plusieurs formes : celle qui correspond aux alternances historiquement les plus régulières $(/ \mathrm{o} / \sim / \varepsilon \mathrm{l} /$ pour les noms en -eau, par exemple); souvent des formes archaïques résultant des apophonies de l'ancien français (/o/ /Ol/ dans CHAPEAU $\rightarrow$ chapelier) et des formes innovantes (/o/ /ot/ dans chapeauter $)$; parfois des formes régionales ou étrangères. C'est normalement le thème $B$ qui constitue le radical des dérivés suffixaux populaires. Mais il est fréquemment altéré par divers procédés (troncation, épenthèse, insertion d'un interfixe, etc.). Le thème B /puiz/ de PUITS, par exemple, conservé tel quel dans le radical /puiz/ de puiser, est augmenté d'une syllabe dans le radical /puizat/ de puisatier, tandis que thème B de ALUMINIUM est accourci par troncation et haplologie dans celui de aluminier.

Pour observer les contraintes phonologiques qui entrent en jeu dans la formation des gentilés, il faut donc s'appuyer à la fois sur le thème $B$ de la base, puisque c'est lui qui peut conditionner le choix du suffixe avec lequel il va entrer en contact, et sur le radical du dérivé, dans la mesure où il a pu être modifié pour être adapté au suffixe. La difficulté est évidemment de déterminer quel est le thème $B$ quand on ne dispose pas de co-dérivés antérieurs. Comme on l'a dit plus haut, il peut être induit le plus souvent à partir de la graphie et l'on a procédé ainsi pour renseigner la base de données. La méthode présente une marge d'incertitude : on ne sait pas toujours, par exemple, si un $-s$ ou un - $x$ final représente une consonne latente ou si elle garde le souvenir d'une marque de flexion qui n'entre pas dans l'alternanace thématique. Compte tenu de l'importance quantitative du corpus, il ne semble pas que cette incertitude soit de nature à invalider les observations qu'on peut en tirer. Dans la pratique, on a admis comme thème B de la base la forme manifestée dans le radical du dérivé chaque fois qu'elle correspondait à une alternance (régulière, archaïque, innovante, régionale) observée dans le lexique pour ce type de finale, et dans le cas contraire on a postulé la plus vraisemblable à partir de la graphie.

\section{Du thème $B$ au radical}

La comparaison entre le thème $B$ de la base et le radical du dérivé n'est pas pertinente pour -ien et -éen puisqu'ils sélectionnent normalement non pas le thème $B$ mais un thème savant. Nous les avons fait figurer quand même dans les tableaux 1 et 2 parce qu'ils sont souvent choisis non pas en fonction du " cahier des charges » (supra, § 1 ) mais parce que la finale de la base rendait indésirables -ais et -ois (et dans ce cas ils suivent généralement le thème populaire). Les autres suffixes ont été regroupés en fonction du timbre de leur voyelle : avec -in(e), les suffixes en /i/ et les très rares gentilés en /y/ ; avec -ain(e), les suffixes en $/ E /$ et en $/ \varnothing /$; etc. ${ }^{6}$ Ont été éliminés les suffixes trop marqués régionalement et davantage susceptibles d'influences étrangères (-ar au Pays Basque, -enc en pays catalan, -asque), et quelques formes très isolées ou douteuses. De ce fait, le total indiqué sur la dernière ligne des tableaux est légèrement supérieur à la somme des lignes précédentes. 


\subsection{Les dérivés en -ais et en -ois : quelle consonne devant le suffixe ?}

Sur la dernière ligne du Tableau 1, on peut voir que dans l'ensemble du corpus 1.1 les sifflantes représentent $11,3 \%$ des accroches consonantiques dans le thème $\mathrm{B}$ de la base et 16,8 \% dans le radical du dérivé (les pourcentages se lisent horizontalement). Dans les lignes supérieures, que ces pourcentages sont de $8,3 \%$ et $8,7 \%$ pour les gentilés en -ois, 3,5 \% et 2,8 \% pour les gentilés en -ais. Cette sous-représentation a une raison simple mise en évidence par Plénat (2008) : la contrainte dissimilative, qui proscrit la consécution à faible distance du même phonème ou de deux phonèmes proches (en l'occurrence l'accroche consonantique et, au féminin, le /z/ du suffixe). L'écart par rapport à la moyenne, cependant, est nettement plus important pour -ais que pour -ois à cause de la constitution complexe du second : le /w/ de -ois introduit une distance supplémentaire entre la sifflante du radical et celle du suffixe, distance qui atténue l'effet de la contrainte dissimilative. Françoise est moins dysphonique que Française. La différence entre -ais et -ois se voit également dans la comparaison entre le chiffre correspondant au thème $\mathrm{B}$ de la base et celui qui correspond au radical du dérivé. Si elle ne s’est pas exercée sur le choix du suffixe, la contrainte dissimilative peut le faire sur la formation du radical. La consonne indésirable peut être remplacée par une autre (7a), éloignée par un interfixe (7b), éliminée par une troncation (7c), par le recours à une base supplétive (7d), ou par une combinaison de plusieurs de ces procédés.
(7a) DAMVIX (85) $\rightarrow$ Damvitais,- aise
(7c) SARCENAS (38) $\rightarrow$ Sarcenais, -aise
(7b) LA FORCE (24) $\rightarrow$ Forcelais, -aise
(7d) BAGNeuX (54) $\rightarrow$ Balnéolais, -aise

\begin{tabular}{|c|c|c|c|c|c|c|c|c|c|c|c|c|c|c|c|c|c|c|}
\hline & \multicolumn{2}{|c|}{$\begin{array}{c}/ \mathrm{p} / / \mathrm{b} / / \mathrm{m} / \\
/ \mathrm{f} / / \mathrm{v} /\end{array}$} & \multicolumn{2}{|c|}{$/ \mathrm{t} / / \mathrm{d} / / \mathrm{n} /$} & \multicolumn{2}{|c|}{$/ \mathrm{s} / / \mathrm{z} /$} & \multicolumn{2}{|c|}{$/ \mathrm{l} / \mathrm{r} /$} & \multicolumn{2}{|c|}{$/ \int / / 3 /$} & \multicolumn{2}{|c|}{$/ \mathrm{j} / / \mathrm{n} / / \mathrm{u} /$} & \multicolumn{2}{|c|}{$/ \mathrm{k} / \mathrm{g} / / \mathrm{w} /$} & \multicolumn{2}{|c|}{ pas de $C$} & \multirow[t]{2}{*}{ total } \\
\hline & & th. B & rad. & th. B & rad. & th. B & rad. & th. B & rad. & th. B & rad. & th. B & rad. & th. B & rad. & th. B & rad. & \\
\hline \multirow{2}{*}{-ois } & $\mathrm{nb}$ & 680 & 702 & 5029 & 5595 & 1208 & 1265 & 3926 & 4025 & 655 & 803 & 1487 & 589 & 1338 & 1585 & 262 & 21 & \\
\hline & $\%$ & 4,7 & 4,8 & 34,5 & 38,4 & 8,3 & 8,7 & 26,9 & 27,6 & 4,5 & 5,5 & 10,2 & 4,0 & 9,2 & 10,9 & 1,8 & 0,1 & \\
\hline \multirow{2}{*}{-ais } & $\mathrm{nb}$ & & 104 & 4038 & 4205 & 259 & 210 & 1586 & 1680 & 85 & 80 & 613 & 516 & 615 & 631 & 119 & 8 & 74 \\
\hline & $\%$ & 1,6 & 1,4 & 54,3 & 56,6 & 3,5 & 2,8 & 21,3 & 22,6 & 1,1 & 1,1 & 8,2 & 6,9 & 8,3 & 8,5 & 1,6 & 0,1 & \\
\hline \multirow{2}{*}{-ien } & $\mathrm{nb}$ & & 383 & 772 & 657 & 1774 & 3376 & 1230 & 1528 & 113 & 164 & 2045 & 117 & 138 & 20 & 265 & 329 & 657 \\
\hline & $\%$ & 3,6 & 5,8 & 11,7 & 10,0 & 27,0 & 51,4 & 18,7 & 23,2 & 1,7 & 2,5 & 31,1 & 1,8 & 2,1 & 0,3 & 4,0 & 5,0 & \\
\hline \multirow[b]{2}{*}{-éen } & $\mathrm{nb}$ & 43 & 133 & 64 & 237 & 101 & 611 & 100 & 310 & 15 & 89 & 468 & 134 & 11 & 7 & 720 & 1 & \\
\hline & $\%$ & 2,8 & 8,7 & 4,2 & 15,6 & 6,6 & 40,1 & 6,6 & 20,4 & 1,0 & 5,8 & 30,7 & 8,8 & 0,7 & 0,5 & 47,3 & 0,1 & \\
\hline \multirow{2}{*}{$\begin{array}{l}\text {-in(e), } \\
\text {-iste, }-u \\
\text { etc. }\end{array}$} & $\mathrm{nb}$ & 74 & 81 & 390 & 601 & 126 & 84 & 197 & 219 & 25 & 20 & 142 & 11 & 52 & 54 & 68 & 4 & $10^{2}$ \\
\hline & $\%$ & 6,9 & 7,5 & 36,3 & 56,0 & 11,7 & 7,8 & 18,3 & 20,4 & 2,3 & 1,9 & 13,2 & 1,0 & 4,8 & 5,0 & 6,3 & 0,4 & 101 \\
\hline \multirow{2}{*}{$\begin{array}{l}\text {-ain(e), } \\
\text {-et, -ier } \\
\text { etc. }\end{array}$} & $\mathrm{nb}$ & 83 & 76 & 538 & 672 & 136 & 83 & 315 & 413 & 24 & 17 & 180 & 60 & 62 & 73 & 75 & 19 & 141 \\
\hline & $\%$ & 5,9 & 5,4 & 38,1 & 47,6 & 9,6 & 5,9 & 22,3 & 29,2 & 1,7 & 1,2 & 12,7 & 4,2 & 4,4 & 5,3 & 5,3 & 1,3 & \\
\hline \multirow{2}{*}{$\begin{array}{l}\text {-at, -ard, } \\
\text {-and } \\
\text { etc. }\end{array}$} & $\mathrm{nb}$ & & 20 & 172 & 138 & 163 & 138 & 188 & 264 & 19 & 21 & 295 & 330 & 23 & 25 & 52 & 4 & 94 \\
\hline & $\%$ & 3,0 & 2,1 & 18,3 & 14,7 & 17,3 & 14,7 & 20,0 & 28,0 & 2,0 & 2,2 & 31,4 & 35,1 & 2,4 & 2,7 & 5,5 & 0,4 & \\
\hline \multirow{2}{*}{$\begin{array}{l}\text {-ot, -aud, } \\
\text {-on } \\
\text { etc. }\end{array}$} & $\mathrm{nb}$ & 24 & 13 & 199 & 155 & 125 & 58 & 195 & 260 & 52 & 68 & 325 & 411 & 33 & 27 & 41 & 2 & $7 y$ \\
\hline & $\%$ & 2,4 & 1,3 & 20,0 & 15,6 & 12,6 & 5,8 & 19,6 & 26,2 & 5,2 & 6,8 & 32,7 & 41,3 & 3,3 & 2,7 & 4,1 & 0,2 & \\
\hline \multirow[b]{2}{*}{-ou } & $\mathrm{nb}$ & 2 & 3 & 21 & 22 & 15 & 11 & 32 & 35 & 3 & 2 & 13 & 16 & 2 & 1 & 3 & 1 & \\
\hline & $\%$ & 2,2 & 3,3 & 23,1 & 24,2 & 16,5 & 12,1 & 35,2 & 38,5 & 3,3 & 2,2 & 14,3 & 17,6 & 2,2 & 1,1 & 3,3 & 1,1 & \\
\hline \multirow{2}{*}{$\begin{array}{l}\text { total } \\
\text { corpus } \\
1.1\end{array}$} & $\mathrm{nb}$ & 1292 & 1518 & 11279 & 12351 & 3931 & 5855 & 7795 & 8761 & 993 & 1268 & 5600 & 2207 & 2281 & 2436 & 1619 & 394 & 3479 \\
\hline & $\%$ & 3,7 & 4,4 & 32,4 & 35,5 & 11,3 & 16,8 & 22,4 & 25,2 & 2,9 & 3,6 & 16,1 & 6,3 & 6,6 & 7,0 & 4,7 & 1,1 & \\
\hline
\end{tabular}

Tableau 1 : Comparaison entre le thème $B$ de la base et le radical du dérivé : l’accroche consonantique 
Une quarantaine de toponymes en -ens ont un gentilé en -ais, mais on ne trouve parmi eux aucun ${ }^{\circ}$-ensais, seulement des dérivés en -enais, -ennais, -anais. Avec -ois, les modifications du radical illustées en (7) sont moins nombreuses. Sur les bases en -ens, 38 (sur 98) conservent la consonne et donnent des dérivés en -ensois. Et les cas où une sifflante est éliminée sont largement compensés par ceux où, au contraire, une sifflante est introduite par une épenthèse (cf. exemple en (9a)). De sorte que, finalement, la proportion des sifflantes devant -ois ne diminue pas et même augmente légèrement.

Un tableau plus détaillé ferait apparaître une autre différence dans l'effet de la contrainte dissimilative : l'écart par rapport à la moyenne est plus important quand la consonne est la sonore /z/, identique à celle du suffixe, moins important quand il s'agit de la sourde /s/. L’opposition de sonorité introduit entre les deux consonnes une autre sorte de distance qui rend moins nécessaire la dissimilation. Sénassaise $(\leftarrow$ SENAS (13)) est moins dysphonique que Esplasaise $(\leftarrow$ ESPLAS (09)).

Dans une autre colonne du Tableau 1, on voit également que les palatales sont, elles aussi, sousreprésentées devant les deux suffixes principaux. La raison en est assez évidente s'agissant de -ois : la consécution immédiate de deux glides n'est pas très heureuse quand le / $\mathrm{w} /$ de -ois doit suivre un $/ \mathrm{j} /$ après voyelle (DREUIL $\rightarrow$ dreuillois), et elle oblige à une diérèse lorsque le yod du thème $\mathrm{B}$ suit une consonne (BRICY $\rightarrow$ Bricyois). Quant au /n/, ou bien il devient /N/ devant -ois, ou bien, suivant les locuteurs, il reste proche de $/ \mathrm{nj} /$, mais dans un cas comme dans l'autre le résultat n'est pas très heureux non plus (FRAGNES $\rightarrow$ Fragnois). On s'explique moins bien que les palatales soient indésirables devant -ais. Un yod, certes, ne fait pas une bonne attaque, mais un dérivé comme Versaillais ne heurte pas l'oreille. Après consonne, le yod est très fréquent et bien accepté dans le lexique général (cf. les nombreux dérivés en -ier ou en -ien). On trouve pourtant très peu de gentilés où -ais suit une accroche en consonne + yod (type MAUNY $\rightarrow$ Maunyais). Quand une base en palatale est néanmoins suffixée en -ois ou en -ais, le yod est souvent éliminé (8a), le /n/ remplacé /n/ (8b), l’un ou l’autre éloigné ou remplacé par un interfixe (8c).
(8a) VIERZY (02) $\rightarrow$ Vierzois, -oise
(8b) Glatigny (60) $\rightarrow$ Glatinois, -oise
SAINTE-EULALIE (24) $\rightarrow$ Eulalais, -aise
(8c) VRIGNY (51) $\rightarrow$ Vrignolais, -aise
LA ROMAGNE (08) $\rightarrow$ Romanais, -aise
ANJOUTEY (90) $\rightarrow$ Anjoutinois, -oise

Ou bien, au lieu de laisser jouer les alternances régulières $/ \mathrm{i} / \sim / \mathrm{j} /$ et $/ \varepsilon / \sim / \varepsilon \mathrm{j} /{ }^{7}$, on conserve la finale vocalique de la forme libre et on lui ajoute une consonne épenthétique (9a). Assez fréquent également, le recours au thème savant lorsqu'il est plus favorable $(9 b)$.
(9a) VERRIE (49) $\rightarrow$ Verrinois, -oise LOUCHY (03) $\rightarrow$ Louchyssois, -oise
(9b) ReILly (60) $\rightarrow$ Reillacois, -oise ${ }^{8}$

\author{
SOUGY (45) $\rightarrow$ Sougycois, -oise \\ CUSSEY (25) $\rightarrow$ Cusseylois, -oise \\ MARIGNY (45) $\rightarrow$ Marignacais, -aise
}

Pour les palatales comme pour les sifflantes, c'est surtout -ien qui hérite des bases dont -ais et -ois ne veulent pas. Avec un effet différent sur le radical des dérivés. Pour les premières, le yod de la base se confondant par haplologie avec celui du suffixe, c'est une autre accroche qui apparaît à la fin du radical (d'où le chiffre très faible sur la ligne correspondante dans le Tableau 1). Pour les secondes, au contraire, l'abondance des sifflantes entraîne, par effet boule de neige, le choix de /z/ comme épenthèse privilégiée. D’où le fait que plus de la moitié des radicaux de gentilés en -ien sont terminés par une sifflante.

Sont également sous-représentés devant -ais et -ois les toponymes dont le thème $\mathrm{B}$ est dépourvu d'accroche consonantique. Comme il s'agit souvent de noms en /e/ sans consonne latente, ils se prêtent tout naturellement à une dérivation en -éen avec haplologie (SEGRÉ (49) $\rightarrow$ Segréen). Pour les autres, l’hiatus est presque toujours évité grâce aux procédés attendus d'épenthèse ${ }^{9}$ ou de troncation.

Les pourcentages supérieurs à la moyenne sont plus difficiles à interpréter. Ils sont en partie l'effet d'un simple équilibrage mécanique par rapport à la moyenne générale (un déficit sur une ligne entraîne automatiquement un surcroît sur les autres). Une différencce assez nette apparaît cependant entre -ais et -ois. Devant le premier, ce sont les dentales qui sont fortement sur-représentées. Devant le second, ce sont les labiales et les vélaires, en accord avec le double caractère du /w/ propre au suffixe -ois. On remarque en particulier un grands nombre d'épenthèses en $/ \mathrm{k} /$ (exemple en (9a)), alors qu'avec -ais il y en a très peu, et des substitutions /t/ > /k/ (SAINT-PRIVAT (19) $\rightarrow$ Saint-Privacois). 


\subsection{Les dérivés en -ais et en -ois : quelle voyelle avant le suffixe ?}

Si l’on observe, maintenant, les voyelles présentes dans la dernière syllabe du thème $\mathrm{B}$ ou du radical (Tableau 2), on constate sans surprise que /E/ est sous-représenté devant -ais et /A/ devant -ois : la contrainte dissimilative s'exerce aussi sur les voyelles. Mais ici encore l'écart par rapport à la moyenne est beaucoup moins important pour -ois que pour -ais : pour les voyelles comme pour les consonnes, l'effet de la contrainte dissimilative s'affaiblit quand les phonèmes identiques sont éloignés par un phonème supplémentaire. Autre différence, également observée avec les sifflantes : la proportion de /A/ devant -ois ne baisse pas (et même augmente légèrement) quand on passe du thème $\mathrm{B}$ de la base au radical du dérivé tandis que le /E/ d'un toponyme sélectionné pour être suffixé en -ais est fréquemment éliminé ou éloigné par les procédés (troncation, interfixation) énumérés plus haut (10a), ou carrément remplacé par une autre voyelle (10b).

(10a) MAISONCELLE (72) $\rightarrow$ Maisonnais, aise
MeX (Sr) $\rightarrow$ Mexannais, aise (10b) BAILLET (95) $\rightarrow$ Baillotais, aise SAINT-GOBAIN (02) $\rightarrow$ Gobanais, -aise

\begin{tabular}{|c|c|c|c|c|c|c|c|c|c|c|c|c|c|c|c|c|c|c|}
\hline & \multicolumn{2}{|c|}{ i/ } & \multicolumn{2}{|c|}{$/ \mathrm{y} /$} & \multicolumn{2}{|c|}{$/ \mathrm{E} / / \tilde{\varepsilon} /$} & \multicolumn{2}{|c|}{ 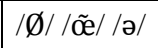 } & \multicolumn{2}{|c|}{$/ \mathrm{A} / / \tilde{\mathrm{a}} /$} & \multicolumn{2}{|c|}{ /0/ /כ̃ / } & \multicolumn{2}{|c|}{$/ \mathrm{u} /$} & \multicolumn{2}{|c|}{ pas de $\mathrm{V}$} & \multirow[b]{2}{*}{ total } \\
\hline & & th. B & rad. & th. B & rad. & th. B & rad. & th. B & rad. & th. B & rad. & th. B & rad. & th. B & rad. & th. B & rad. & \\
\hline \multirow[b]{2}{*}{-ois } & nb & 2827 & 3626 & 392 & 383 & 5370 & 4633 & 631 & 521 & 2788 & 2855 & 1656 & 1685 & 908 & 882 & 13 & 0 & 458 \\
\hline & $\%$ & 19,4 & 24,9 & 2,5 & 2,6 & 36,8 & 31,8 & 4,3 & 3,6 & 19,1 & 19,6 & 11,4 & 11,6 & 6,2 & 6,0 & 0,1 & 0,0 & \\
\hline \multirow[b]{2}{*}{-ais } & $\mathrm{nl}$ & 1015 & 1031 & 62 & 64 & 803 & 625 & 261 & 253 & 2697 & 2762 & 2437 & 2532 & 158 & 166 & 1 & 1 & 74 \\
\hline & $\%$ & 13,7 & 13,9 & 0,8 & 0,9 & 10,8 & 8,4 & 3,5 & 3,4 & 36,3 & 37,2 & 32,8 & 34,1 & 2,1 & 2,2 & 0,0 & 0,0 & \\
\hline \multirow{2}{*}{-ien } & nb & 987 & 1241 & 223 & 458 & 2230 & 1913 & 451 & 208 & 1499 & 1522 & 740 & 964 & 436 & 266 & 8 & 1 & $65 ?$ \\
\hline & $\%$ & 15,0 & 18,9 & 3,4 & 7,0 & 33,9 & 29,1 & 6,9 & 3,2 & 22,8 & 23,2 & 11,3 & 14,7 & 6,6 & 4,0 & 0,1 & 0,0 & \\
\hline \multirow{2}{*}{-éen } & nb & & 242 & & 145 & 1061 & 224 & 42 & 49 & 177 & 487 & 87 & 282 & 47 & 80 & 1 & 13 & 152 \\
\hline & $\%$ & 4,1 & 15,9 & 2,9 & 9,5 & 69,5 & 14,9 & 2,8 & 3,2 & 11,6 & 32,0 & 5,7 & 18,5 & 3,1 & 5,3 & 0,1 & 0,9 & \\
\hline \multirow{2}{*}{$\begin{array}{l}\text {-in(e), } \\
\text {-iste, -u } \\
\text { etc. }\end{array}$} & nb & 42 & 44 & 25 & 33 & 312 & 182 & 73 & 38 & 327 & 344 & 215 & 350 & 80 & 83 & 0 & 0 & 107 \\
\hline & $\%$ & 3,9 & 4,1 & 2,3 & 3,1 & 29,1 & 17,0 & 6,8 & 3,5 & 30,4 & 32,0 & 20,0 & 32,6 & 7,4 & 7,7 & 0,0 & 0,0 & 101 \\
\hline \multirow{2}{*}{$\begin{array}{c}\text {-ain(e), } \\
\text {-et, -ier } \\
\text { etc. }\end{array}$} & nb & 116 & 178 & 34 & 39 & 412 & 288 & 104 & 84 & 327 & 293 & 322 & 434 & 95 & 96 & & 1 & 14 \\
\hline & $\%$ & 8,2 & 12,6 & 2,4 & 2,8 & 29,2 & 20,4 & 7,4 & 5,9 & 23,1 & 20,7 & 22,8 & 30,7 & 6,7 & 6,8 & 0,2 & 0,1 & \\
\hline \multirow{2}{*}{$\begin{array}{l}\text {-at, -ard, } \\
\text {-and } \\
\text { etc. }\end{array}$} & nb & 128 & 163 & & 34 & 299 & 260 & & 60 & 172 & 155 & & 205 & 60 & 62 & 2 & 1 & 94 \\
\hline & $\%$ & 13,6 & 17,3 & 2,9 & 3,6 & 31,8 & 27,7 & 8,8 & 6,4 & 18,3 & 16,5 & 18,0 & 21,8 & 6,4 & 6,6 & 0,2 & 0,1 & \\
\hline \multirow{2}{*}{$\begin{array}{l}\text {-ot, -aud, } \\
\text {-on } \\
\text { etc. }\end{array}$} & nb & 158 & 268 & 27 & 37 & 324 & 206 & 59 & 63 & 274 & 284 & 102 & 78 & 48 & 57 & 2 & 1 & \\
\hline & $\%$ & 15,9 & 27,0 & 2,7 & 3,7 & 32,6 & 20,7 & 5,9 & 6,3 & 27,6 & 28,6 & 10,3 & 7,8 & 4,8 & 5,7 & 0,2 & 0,1 & \\
\hline \multirow[b]{2}{*}{-OU } & nb & & 10 & 0 & 0 & 34 & 30 & 11 & 11 & 37 & 35 & 4 & 1 & 2 & 3 & 0 & 1 & 9 \\
\hline & $\%$ & 3,3 & 11,0 & 0,0 & 0,0 & 37,4 & 33,0 & 12,1 & 12,1 & 40,7 & 38,5 & 4,4 & 1,1 & 2,2 & 3,3 & 0,0 & 1,1 & \\
\hline \multirow{2}{*}{$\begin{array}{l}\text { total } \\
\text { corpus } \\
1.1\end{array}$} & nb & 5349 & 6821 & 840 & 1201 & 10886 & 8394 & 1721 & 1287 & 8363 & 8798 & 5755 & 6562 & 1845 & 1706 & 31 & 20 & \\
\hline & $\%$ & 15,4 & 19,6 & 2,4 & 3,5 & 31,3 & 24,1 & 4,9 & 3,7 & 24,0 & 25,3 & 16,5 & 18,9 & 5,3 & 4,9 & 0,1 & 0,1 & \\
\hline
\end{tabular}

Tableau 2 : Comparaison entre le thème $B$ de la base et le radical du dérivé : la dernière voyelle

Ces substitutions se font rarement au profit de /i/, beaucoup plus souvent au profit de /O/ ou de /A/. D’une façon générale, on constate que les autres voyelles d'avant sont également sous-représentées devant -ais alors que /A/ et /O/ sont fortement sur-représentées. En plus des substitutions /E/ > /O/ et /E/ > /A/, on 
observe des substitutions /i/ > /0/ et /i/ > /A/ (11), comme s’il s'agissait non seulement d'éviter la consécution de deux voyelles identiques mais de maximiser l'écart entre les voyelles qui se suivent.
(11) LE FAVRIL (27) $\rightarrow$ Faverollais, -aise
VILLARLURIN (73) $\rightarrow$ Villarlurannais, -aise

Symétriquement, /0/ est sous-représenté devant -ois, avec un écart par rapport à la moyenne encore plus important que pour /A/, tandis que les voyelles d'avant sont sur-représentées. Et parmi elles, c'est /i/ qui bénéficie des modifications apportées au thème $B$ pour former le radical. Non seulement il remplace des /A/ ou des /0/, directement (12a) ou par l'intermédiaire d'un interfixe (12b), mais il se substitue aussi fréquemment à /E/, directement (12c) ou par l'intermédiaire d'un interfixe (12d). Ici encore, l'écart entre les deux voyelles s'en trouve maximisé.
(12a) Menerval (76) $\rightarrow$ Ménervillois, -oise SAXI-BOURDON (56) $\rightarrow$ Saxibordinois
(12c) BOUCHET (43) $\rightarrow$ Bouchitois, -oise SAINT-ROMAIN (69) $\rightarrow$ Saint-Rominois

\author{
(12b) SAINT-LAURENT (69) $\rightarrow$ Laurentinois \\ CORN (46) $\rightarrow$ Cornicquois, -oise \\ (12d) SAINT-MARCEL (01) $\rightarrow$ Marcellinois \\ CORTAMBERT (71) $\rightarrow$ Cortambertinois
}

Exemple caractéristique de ces altérations : les gentilés construits sur des toponymes en -ain(e)(s). Ils ont normalement un radical en $/ \varepsilon \mathrm{n} /$, comme le thème $\mathrm{B}$ de leur base. Or, sur 68 dérivés en -ais, on trouve 29 finales en -anais, 5 en -inais (et 6 finales diverses) à côté des 28 finales en -ainais qui respectent la contrainte de fidélité. Sur 261 dérivés en -ois, 158 finales en -inois, 23 en -anois, 19 en -enois et seulement 55 en -ainois (et 6 finales diverses). La proportion -ais / -ois, comme on pouvait s'y attendre pour une base en /E/, est plus nettement en faveur de -ois que dans l'ensemble du corpus (où elle est grosso modo de 1 pour 2). Mais ce sont surtout les modifications apportées au radical qui sont intéressantes. Notons d'abord qu'elles affectent une proportion considérable des dérivations (trois sur quatre). Deux d'entre elles peuvent avoir une explication autre que phonologique. Les finales en -anais n'étonnent pas a priori, puisque l'allomorphie $/ \tilde{\varepsilon} / \sim$ /an/ est assez familière. Mais elle est propre, normalement, au vocabulaire savant et devant -ais c'est le thème présuffixal populaire qui est requis. Les bases en -ain(e), d'autre part, ont pu subir l'influence des bases en -in(e), homophones au masculin et plus nombreuses. On sait qu'une alternance plus fréquente (en l'occurrence $/ \tilde{\varepsilon} / \sim /$ in/) a tendance à se substituer à une alternance qui l'est moins (en l'occurrence $/ \tilde{\varepsilon} / \sim / \varepsilon n /$ ). Mais ce facteur, comme le précédent, devrait jouer de la même manière devant -ais et devant -ois, ce qui n'est pas le cas. C'est bien le timbre de la voyelle du suffixe qui conditionne le choix de la voyelle qui précède : /a/ devant / $/$ / et /i/ devant /a/. Le même SAINT-GERMAIN-DUBEL-AIR (46) hésite entre Saint-Germanais et Saint-Germinois.

Le choix des interfixes est également révélateur. On sait (cf. Roché, 2015) qu’ils ont pour raison d’être de satisfaire une contrainte lexicale (en renforçant l'intégration dans une série dérivationnelle grâce à une homophonie en amont du suffixe) ou, plus souvent, une contrainte phonologique - prosodique ou/et segmentale - en ajoutant une syllabe au radical et en modifiant l'accroche offerte au suffixe. Sémantiquement neutres, ils sont choisis en fonction de leur constitution phonologique. Devant -ais, les interfixes les plus fréquents sont -ol-, -on-, -an- et, d'une façon générale, les interfixes en /0/ (63) et en /A/ (27). La rareté des interfixes en /E/ (3) était évidemment prévisible mais ceux en /i/ sont également peu nombreux (9). Devant -ois, on n'attendait pas d'interfixes en /A/ mais ceux en /O/ sont encore moins nombreux. Globalement, si l'on met à part ceux en chwa ${ }^{10}$, $83 \%$ ont une voyelle d'avant, $17 \%$ un /A/ ou un /0/.

Parmi les voyelles, on a laissé à part le cas de /u/, après lequel la sur-représentation de -ois est due au très grand nombre de toponymes en -bourg et surtout en -court qui donnent des dérivés en -bourgeois et -courtois pour une raison lexicale (supra, § 1). En dehors de ces deux sous-séries, la répartition de -ais et de -ois après /u/ est identique à celle qu’on observe dans l'ensemble du corpus.

\subsection{Autres suffixes : les dérivés en /i/,/y/,/E/, / $\tilde{\varepsilon} /, / \varnothing /$}

Malgré les particularités de chaque suffixe, le tableau présenté par les gentilés construits avec les autres suffixes comportant une voyelle d'avant est assez cohérent et très semblable à celui des dérivés en -ais. Du côté des voyelles, les /E/ sont rares devant -ain(e) et les autres suffixes en /E/, comme on s'y attendait, et les /i/ encore plus rares devant -in(e) et les autres suffixes en /i/. Mais les /E/ sont aussi en proportion inférieure à la moyenne devant les suffixes en /i/, et les /i/ devant les suffixes en /E/, les bénéficiaires de cette défaveur étant /A/ et /0/. L’interfixe le plus sollicité est, de loin, -ot- et, en dehors de ceux en chwa, 
neuf sur dix ont pour voyelle /0/ ou /A/. Ceux en /E/ sont rares même devant -in(e)), ceux en /i/ rares même devant -ain(e).

Du côté des consonnes, la répartition est également semblable à celle qu’on trouve devant -ais et l'on ne s'intéressera qu'aux thèmes en yod, devant les suffixes en /i/ puis devant ceux en /E/. Les palatales sont encore relativement nombreuses dans le thème $\mathrm{B}$ des bases qui reçoivent ces suffixes, mais dans le radical des dérivés en $/ \mathrm{i} /$ ne restent que quatre $/ \mathrm{h} /$, quatre $/ \mathrm{u} /$ et quatre $/ \mathrm{j} /$. Les yods ont disparu régulièrement par haplologie ${ }^{11}$ quand ils suivaient une consonne (thème $B$ des toponymes en $-y$ ou en -ie après consonne). Les exemples illustrés en (13a), cependant, sont en fait assez rares : dans la majorité des cas, l'élimination du yod n'est pas due à cet effet mécanique mais à une troncation et/ou à l'insertion d'un interfixe (13b).

$$
\begin{aligned}
& \text { (13a) AuBIE (33) } \rightarrow \text { Aubin, -ine } \\
& \text { LoCTUDY (29) } \rightarrow \text { Loctudyste }
\end{aligned}
$$

(13b) FLEURY (71) $\rightarrow$ Fleurandin, -ine MESSERY (74) $\rightarrow$ Messerolin, -ine

Quand le yod résulte d'un ancien $/ K /$ et suit une voyelle, il peut être suivi de $/ \mathrm{i} /$ et il l'est effectivement dans les nombreux toponymes en -illy, -ailly, -ouilly, -euilly. Au thème $\mathrm{B}$, le /i/ final est absorbé par le yod qui le précède et les gentilés correspondant à ces toponymes pourraient être formés directement avec -in(e) ou un autre suffixe en /i/. Or le cas de figure représenté en (14a) est rarissime (trois exemples, dont un au moins est douteux). Dans la plupart des cas, un interfixe vient s'intercaler entre le thème $B$ et le suffixe (14b).

$$
\begin{aligned}
& \text { (14a) Eguilles (13) } \rightarrow \text { Eguillin, -ine } \\
& \text { Huilly ( 71) } \rightarrow \text { Huillyte }
\end{aligned}
$$

(14b) TILLY (35) $\rightarrow$ Tillotin, -ine DROUILly (51) $\rightarrow$ Drouillotin, -ine

Restent les toponymes en -ay, -ey, -oy, eux aussi très nombreux. Leur thème $\mathrm{B}$ est aussi en $/ \mathrm{Vj} /$ et ne se distingue pas des précédents, phonétiquement, en français contemporain. Mais les séquences $/ \mathrm{Vj} /$ de ce type sont très rarement suivies de /i/ dans le lexique général (seuls exemples dans le Grand Robert : essayiste, fédayin, himalayisme) et aucune ne l'est dans notre corpus de gentilés. Ici encore, la difficulté est tournée le plus souvent grâce à un interfixe, qui cette fois se substitue à la finale (16a), ou simplement par une troncation (15b).

$$
\text { (15a) Chailley (89) } \rightarrow \text { Chaillotin, -ine }
$$

$$
\text { (15b) BugEY (F) } \rightarrow \text { Bugiste }
$$

Le plus remarquable, nous semble-t-il, avec ces finales problématiques en yod, est qu'elles n'empêchent pas une suffixation en /i/ (39 toponymes en -ay(e) ou -ey sont ainsi suffixés) mais qu'elles conduisent à un aménagement du radical qui affecte à la fois l'accroche consonantique et la voyelle qui précède pour les mettre en harmonie avec le suffixe.

Devant les suffixes en /E/, les accroches en yod ne posent pas les mêmes problèmes, mais elles sont quand même en nombre inférieur à la moyenne et éliminées deux fois sur trois par les procédés déjà décrits de troncation, épenthèse, interfixation (16), qui ici encore peuvent se combiner.

$$
\begin{aligned}
& \text { (16) LIZERAY (36) } \rightarrow \text { Lizerain, -aine } \\
& \text { Plemy (22) } \rightarrow \text { Plémytain, -aine }
\end{aligned}
$$

$$
\begin{aligned}
& \text { LA BATHIE (73) } \rightarrow \text { Bathiolain, -aine } \\
& \text { JOUY (28) } \rightarrow \text { Jouysotier, -ière }
\end{aligned}
$$

Le suffixe -ier, grâce à son yod, permettait un traitement particulier, par l'haplologie, des accroches en yod, mais cette possibilité n'est utilisée que par trois VITRY, qui donnent deux Vitrier et un Vitryer, et par l'exemple en (17a). Dans les autres gentilés en -ier (une vingtaine après palatale), on retrouve les mêmes procédés que précédemment (17b).

$$
\text { (17a) Menotey (39) } \rightarrow \text { Menoteyer, -ère }
$$

(17b) QUINCEY (21) $\rightarrow$ Quinçonnier, -ière

\subsection{Autres suffixes : les dérivés en $/ 0 /, / \tilde{J} /, / \mathrm{A} /, / \tilde{a} /$}

Les gentilés formés avec les suffixes en /0/ et en /A/ contrastent fortement avec les précédents. Comme dans ceux en -ois, les voyelles d'avant y sont plus nombreuses, dans la syllabe qui précède le suffixe, que les voyelles d'arrière (parmi lesquelles nous rangerons $/ \mathrm{A} /{ }^{12}$ ). La contrainte dissimilative, ici encore, fait qu'il y a peu de /O/ devant -ot et -on, peu de /A/ devant -ard et -at. Soit parce que ces suffixes n'ont pas été choisis pour succéder à une voyelle identique à la leur, soit parce que la voyelle identique a été remplacée par une autre (18a) ou éloignée grâce à un interfixe (18b). 


\section{(18a) BouRgogne $\rightarrow$ Bourguignon, -onne $\quad$ (18b) CORME (17) $\rightarrow$ Cormillon, -onne}

Mais on observe fréquemment qu'un /i/ ou un / $\tilde{\varepsilon} /$ se substitue à un /A/ devant /0/ ou à un /0/ devant /A/ (19a) ; ou qu'un /O/ soit éloigné d'un /A/ et un /A/ d'un /0/ par l'intermédiaire d'un interfixe (19b). Des altérations, par conséquent, qui n’ont pas pour objet d'éviter la répétition d’une même voyelle mais d'augmenter l'écart entre deux voyelles successives.
(19a) MARBACHE (54) $\rightarrow$ Marbichon, -onne GRANGES (88) $\rightarrow$ Gringeaud, -aude ROMONT (Sr) $\rightarrow$ Rominat, -ate CAPLAN (Q) $\rightarrow$ Caplinot, -ote

\author{
(19b) SABRES (40) $\rightarrow$ Sabringot, -ote \\ LA GARDE (38) $\rightarrow$ Gardillon, -onne \\ COMBREs (42) $\rightarrow$ Combrisard, -arde \\ ORMES (71) $\rightarrow$ Ormillat, -ate
}

La contrainte dissimilative, d'autre part, est neutralisée, et une voyelle identique à celle du suffixe est bien tolérée, si entre elles s’intercale un /j/ ou un /n/, présent dans la base (20a) ou introduit par une épenthèse (20b) ou une substitution (20c).

\section{(20a) MANDAilles (15) $\rightarrow$ Mandaillard, -arde Les Amognes (F) $\rightarrow$ Amognot, -ote}

(20b) GLANES (46) $\rightarrow$ Glaniat, -ate

(20c) CHAMBON (41) $\rightarrow$ Chambognot, -ote

Une voyelle identique à celle du suffixe est également bien tolérée si entre les deux s'intercale un interfixe en chwa + liquide (21). Du fait de l'amuïssement du chwa, deux voyelles identiques (ou identiques à la nasalité près) se suivent dans deux syllabes successives, mais le complexe consonantique qui les sépare introduit une distance suffisante pour désamorcer, semble-t-il, la contrainte dissimilative.
(21) BEAUCE (F) $\rightarrow$ Beauceron, -onne
PASSY (74) $\rightarrow$ Passerand, -ande

Et comme on l'avait observé pour -ois, parmi les voyelles d'avant, la proportion des voyelles hautes augmente sensiblement au détriment des voyelles moyennes quand on passe du thème $\mathrm{B}$ de la base au radical du dérivé. Dans de nombreux gentilés, un /E/ est remplacé par un /i/ ou éloigné du suffixe par un interfixe en /i/ (22).
(22) HuEZ (38) $\rightarrow$ Huizat, -ate SAINTE-MADELEINE (Q) $\rightarrow$ Madelinot, -ote
VOUVRAY (37) $\rightarrow$ Vouvrillon, -onne BOUERE (53) $\rightarrow$ Bouérillot, -ote

Du côté des consonnes, une différence importante oppose ces gentilés à ceux qui sont formés avec -ois : la proportion considérable d'accroches en palatale (autour de 32 \% dans le thème $\mathrm{B}$ de la base, 35,1 \% dans le radical des dérivés en /A/, 41,3 \% devant ceux en /0/). Le premier chiffre s'explique en partie par la sous-représentation de -ais et de -ois après ce type d'accroche : les autres suffixes jouent les remplaçants. Et dans ce cas, les alternances régulières $/ \mathrm{i} / \sim / \mathrm{j} /$ et $/ \mathrm{ji} / \sim \mathrm{j} /$ sont presque toujours respectées (23).
(23) SACY (89) $\rightarrow$ Sacyat, -ate
BRIE $(\mathrm{F}) \rightarrow$ Briard, -ardre
BARBY (08) $\rightarrow$ Barbyon, -onne
JUVIGNY (51) $\rightarrow$ Juvignot, -ote

Mais si le second chiffre est supérieur au premier, c’est que, en plus des palatales présentes dans le thème $\mathrm{B}$ qui sont conservées dans le radical du dérivé, d'autres ont été ajoutées à des bases qui n’en comportaient pas. On observe ainsi un nombre important d'épenthèses (24a) et d'interfixations (24b) en yod, et dans une moindre mesure en /n/ (24c).
(24a) ChePpes (51) $\rightarrow$ Cheppiat, -ate ORCHAISE (41) $\rightarrow$ Orchaisiot, -ote
(24c) SAVY (01) $\rightarrow$ Savignon, -onne

(24b) BRON (69) $\rightarrow$ Brondillant, -ante LA GARDE (38) $\rightarrow$ Gardillon, -onne

La fréquence des yods devant les suffixes en /0/, en particulier -eau et -aud, a fini par donner un suffixe plus ou moins autonome -iau, dont l'exemple le plus connu est Morvandiau ${ }^{13}$. On observe aussi de nombreux cas où un yod se substitue à une autre consonne (25a), en particulier des substitutions $/ \mathrm{l} />/ \mathrm{K} /$ et $/ \mathrm{n} /$ $>/ \mathrm{n} /$ (25b), inverses de celles qui ont été notées devant -ais, et qui se combinent avec les substitutions /E/ $>$ /i/ déjà mentionnées. Ainsi que de curieuses métathèses qui déplacent un yod pour le mettre au contact du suffixe (25c).

(25a) Montgueux (10) $\rightarrow$ Montgueuillat -ate

(25b) VERPEL (08) $\rightarrow$ Verpillart, -arte

(25c) Cumieres (51) $\rightarrow$ Cumariot, -ote
Droupt (10) $\rightarrow$ Drouillon -onne POUlaines (36) $\rightarrow$ Poulignot, -otte FENIOUX (17) $\rightarrow$ Fenouillard, -arde 
Il y a donc une raison positive à ces aménagements du radical : si un yod est indésirable devant -ois, il est le bienvenu devant -ard et -and et encore plus devant -ot et -on.

\subsection{Autres suffixes : les dérivés en / $\mathrm{u} /$}

Les gentilés en -ou ${ }^{14}$ sont peu nombreux (une centaine) et de ce fait les pourcentages qu'on peut calculer sont moins significatifs. Mais il faut les mettre à part parce qu'ils se distinguent assez nettement des deux ensembles précédents. Comme pour les gentilés en / $/$ / et en / $\mathrm{A} /$, la dernière voyelle du radical est majoritairement une voyelle d'avant (dans les mêmes proportions). Mais cette voyelle est beaucoup moins souvent un /i/, quatre fois sur cinq une voyelle moyenne. Parmi les autres voyelles, les /u/ sont évidemment très peu nombreux, mais les $/ 0 /$ ne le sont guère davantage. Presque toutes sont des $/ \mathrm{A} /$. Les voyelles les moins représentées sont donc les voyelles les plus proches, soit par leur point d'articulation (/0/), soit par leur aperture (/i/ et /y/, ce dernier totalement absent).

Du côté des consonnes, le fait le plus saillant pourrait être la sur-représentation des liquides, mais on ne voit pas quelle signification particulière on pourrait lui donner. En revanche, le nombre relativement important de palatales rapproche ces dérivés en /u/ de ceux en /0/ et en /A/.

\section{Essai d'interprétation}

Le détour par les autres suffixes confirme et complète les observations que nous avions faites sur les deux principaux, -ois et -ais, et les corrige sur un point essentiel. Comme -ais, les suffixes dont la voyelle est une voyelle d'avant ont une préférence pour les radicaux dont la dernière voyelle est un /A/ ou un $/ 0 /$. Comme -ois, les suffixes dont la voyelle est un /A/ ou un /0/ ont une préférence pour les radicaux dont la dernière syllabe est une voyelle d'avant, et plutôt une voyelle haute qu'une voyelle moyenne. Mais, à la différence de -ois, ces suffixes (-ot, -on -at,- ard...) ont une prédilection marquée pour une accroche en palatale tandis que les suffixes dont la voyelle est une voyelle d'avant, comme -ais, ne l'apprécient pas. Ce sont donc les relations entre voyelles et la question des palatales - du yod essentiellement - qu'il s’agit maintenant de préciser, en les rapprochant et en les éclairant mutuellement.

\subsection{Les relations entre voyelles et la question du yod}

Les suffixes en / $/$ et en /A/, à l'exception notable de -ois, privilégient à la fois une accroche en palatale et une voyelle haute d'avant dans la syllabe précédente. Ces deux préférences sont liées. Les voyelles concernées sont /i/ et /y/, et les accroches consonantiques en palatale sont ou bien les glides correspondant à ces deux voyelles, ou bien des /n/ qui se confondent pratiquement avec /n/ + yod. L'essentiel, par conséquent, se joue autour de $/ \mathrm{i} /$ et de $/ \mathrm{j} /{ }^{15}$. Au contact du suffixe ou dans la syllabe qui le précède, $/ \mathrm{j} /$ et /i/ jouent logiquement le même rôle quant à l'harmonie finale du dérivé puisqu'ils partagent la plupart de leurs traits. Il y a une continuité, et une grande similitude des effets produits, entre l'épenthèse d'un yod (26a), la même avec diérèse (26b), un interfixe en /ij/ (26c) et un autre interfixe en /i/ (26c).
(26a) Pleurs (51) $\rightarrow$ Pleuriot, -ote
(26b) MANDRES (94) $\rightarrow$ Mandrion, -onne
(26c) ARCES (17) $\rightarrow$ Arcillon, -onne
(26d) TRADES (69) $\rightarrow$ Tradirot, -ote

Le rapprochement entre accroche en palatale et voyelle haute d'avant dans la syllabe précédente est faussé pour -ois à cause du glide qu'il contient et qui rend difficile l'enchaînement avec un autre glide (ou avec $/ \mathrm{n} /$ ). Il est faussé, d'une autre façon, pour les suffixes en /i/ à cause des phénomènes d'haplologie. Il faut mettre à part, par conséquent, ces deux cas de figure. Mais pour les autres le contraste est saisissant entre les suffixes en /E/ (dont -ais) d'un côté, et les suffixes en /0/ ou en /A/ de l'autre. Si l'on additionne accroche en palatale et voyelle haute d'avant dans la syllabe précédente ${ }^{16}$, cet ensemble représente les pourcentages suivants :

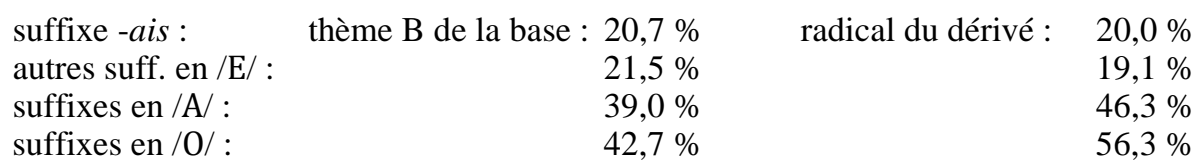




\subsection{Les relations entre voyelles : la dissimilation contre la disharmonie}

Le corpus de gentilés confirme les nombreuses observations de Plénat (2008, 2015, entre autres) sur la contrainte dissimilative dans son effet le plus connu : éviter la consécution à faible distance du même phonème ou de deux phonèmes proches. C'est elle qui est responsable de la rareté des /E/ dans la syllabe précédant -ais ou -ain, des /A/ devant -ois, -at, -ard, des /0/ devant -ot et -on, etc., comme elle évite les accroches en sifflante devant le /z/ de -oise et de -aise. Il s'agit d'une contrainte négative qui vise à éviter un effet dysphonique. C'est la raison pour laquelle, d'une façon générale, elle est surtout active pour éviter les rencontres de consonnes, qui heurtent davantage l'oreille que celles des voyelles (tous les exemples développés par Plénat (2015) à propos de la contrainte dissimilative en français concernent les consonnes). C’est aussi pourquoi son effet s’atténue rapidement quand la proximité ou la similitude des phonèmes concernés diminue. Elle fonctionne à plein lorsque deux phonèmes identiques risquent de se suivre en n'étant séparés que par un seul phonème. C’est le cas, parmi les consonnes, quand le suffixe -ais(e) suit une accroche en /z/. On pourrait observer la même chose pour /t/ devant -ot, pour /n/ devant -in, etc. (ces cas de figure n’ont pas été développés parce qu'ils n'auraient rien apporté de nouveau). Elle est moins vigoureuse lorsqu'une certaine distance s'introduit entre les deux phonèmes. Soit parce que, tout en étant très proches, ils ne sont pas identiques (accroche en $/ \mathrm{s} /$ et non en $/ \mathrm{z} / \mathrm{devant}-\operatorname{ais}(e)$ ). Soit parce qu'un phonème supplémentaire s'introduit entre les deux phonèmes identiques : le $/ \mathrm{w} / \mathrm{de}$-ois fait que les sifflantes sont écartées moins sévèrement devant ce suffixe que devant -ais, et que /A/ est écarté moins sévèrement devant -ois que /E/ devant -ais. Devant les autres suffixes, on a observé également que l'introduction d'une consonne épenthétique ou d'un interfixe en chwa (qui équivalent à remplacer une consonne simple par une attaque branchante ou à ajouter une consonne en coda) désamorcent, en quelque sorte, la contrainte dissimilative. Il est plus difficile de mesurer l'effet des écarts de timbre entre les voyelles à cause des alternances en genre qui opposent, pour un même suffixe, mi-fermée à mi-ouverte (-ot -ote) ou nasale à orale (-ain -aine, -on -onne). Pour cette raison, nous avons neutralisé ces oppositions et considéré que la contrainte dissimilative affecte, globalement, des $/ 0 /$, des $/ \mathrm{E} /$, etc., comme pour les consonnes on a dit globalement qu'elle affecte les sifflantes. La différence entre /o/ et / $/ \mathrm{/}$, ou / $/ \mathrm{J}$ et / $\tilde{\jmath} /$ est comparable à celle qui sépare $/ \mathrm{s} / \mathrm{de} / \mathrm{z} /$.

\subsection{Les relations entre voyelles : la dissimilation pour l'harmonie}

Nous avons vu également que la construction des gentilés tend à favoriser un contraste, un écart maximum entre la dernière voyelle du radical et celle du suffixe. Si l'une est une voyelle haute d'avant, l'autre sera de préférence une voyelle d'arrière, moyenne ou basse. Et vice-versa. C'est donc également une forme de dissimilation puisque le dissemblable est privilégié. Mais d'une façon bien différente et pour une autre raison. Il ne s'agit plus d'éviter ce qui est dysphonique mais de favoriser ce qui est perçu comme plus harmonieux. Une raison positive, par conséquent. On ne peut pas dire que la consécution, dans deux syllabes successives, d'un /A/ et d'un /O/, ou d'un /i/ et d'un /E/, soit désagréable à l'oreille mais il semble préférable, du point de vue de l'harmonie, que le /i/ soit suivi d'un /0/, par exemple, et le /O/ d'un /i/.

Il s'agit bien d'harmonie, en effet : de la recherche, parmi plusieurs suites de sons, de celle qui sera la plus agréable à l'oreille. Et, comme en peinture il y a des harmonies en camaïeu et des harmonies fondées sur les oppositions de couleurs, les langues peuvent favoriser soit les nuances à l'intérieur d'une même gamme, soit les contrastes. D'après nos observations, et pour ce qui concerne les voyelles, le français penche pour la deuxième option, au contraire des langues qui privilégient et parfois imposent une harmonie fondée sur l’homogénéité d’un même registre (arrondi vs écarté, par exemple).

Restent deux questions. Une, secondaire, de métalangue : comment nommer cette contrainte ? «Contrainte d’harmonie », puisque c’est cela qu'elle vise ? Mais « harmonie vocalique » a été consacré pour la contrainte qui vise à la similitude, comme si c'était la seule forme d’harmonie. Il faudrait pouvoir la subdiviser en " harmonie homogène » et " harmonie constrastive ». "Contrainte dissimilative », puisqu'elle vise le dissemblable ? Mais cette expression risque de n’évoquer que l'autre face de la dissimilation, la plus familière. Il faudrait, là aussi distinguer dissimilation positive et dissimilation négative. 
L'autre question est plus fondamentale : quel est le contenu exact de cette contrainte ? Elle n'oppose pas exactement les voyelles d'avant aux voyelles d'arrière mais les voyelles d'avant (hautes et moyennes) à /0/ et à /A/. Et surtout /i/ à /0/, opposition qui mobilise le plus grand nombre de traits. Les oppositions haut/bas et avant/arrière entrent l'une et l'autre en jeu, mais nous ne sommes pas en mesure de déterminer si l'une est plus déterminante que l'autre. Les dérivés en /u/, malgré leur petit nombre, donnent cependant une indication intéressante. On a vu que dans leur radical les voyelles les moins représentées sont les plus proches, soit par leur point d'articulation (/0/), soit par leur aperture (/i/), et les plus représentées /E/ et /A/. Les deux critères semblent donc impliqués. On aimerait également savoir si l'opposition écarté vs arrondi y joue un rôle. Il faudrait pour cela pouvoir distinguer /y/ de /i/ et / Ø/ de /E/. Mais le petit nombre de radicaux en $/ y /$ et en $/ \varnothing /$, et surtout l'extrême rareté des suffixes en $/ y /$ et en $/ \varnothing /$ ne le permet pas.

\subsection{L'harmonie entre consonne et voyelle}

Autre forme d'accord : celui qui harmonise la voyelle du suffixe (ou le complexe glide + voyelle, dans le cas de -ois) avec l'accroche consonantique offerte par le radical du dérivé. Nous avons observé, comme Plénat (2008), que les labiales et les vélaires sont sur-représentées devant -ois, dont le /w/ présente ce double caractère. Tantôt la présence de ces consonnes à l'accroche du thème $B$ de la base a orienté le choix du suffixe vers -ois, tantôt une telle accroche s'est substituée, dans le radical du dérivé, à celle qu'offrait la base. Devant -ais, ce sont les dentales qui sont fortement sur-représentées, plus précisément /t/, /d/ et /n/ puisque /s/ et /z/ sont éliminées à cause de la sifflante du suffixe. Elles le sont également devant les autres suffixes en /E/ et devant ceux en /i/, de façon plus frappante encore si l'on compare le radical du dérivé au thème $\mathrm{B}$ de la base, alors que la consonne du suffixe est le plus souvent un /n/. On trouve également devant ces suffixes une proportion de vélaires relativement importante. Mais ces consonnes sont soit des /w/ par semi-vocalisation (BIzOU (61) $\rightarrow$ Bizouin, SAINT-MALO (35) $\rightarrow$ Malouin), soit des $/ \mathrm{k} /$ appelés par une raison lexicale (SAINT-AFFRIQUE (12) $\rightarrow$ Saint-Affricain), soit des $/ \mathrm{k} /$ ou des /g/ qui suivent des /O/ ou des /A/ (MEDOC $\rightarrow$ Médocain, BoussAC (23) $\rightarrow$ Boussaquin), l'harmonie (constrastive) entre les voyelles l'ayant emporté sur l'harmonie entre consonne et voyelle.

Devant les suffixes en /O/ ou en /A/, la sous-représenation des dentales est frappante : elles sont deux fois moins nombreuses que devant les suffixes en /i/ ou en /E/, alors que les consonnes des suffixes, actualisées au féminin, sont les mêmes que pour les suffixes précédents. Ce ne sont pas, par conséquent, des raisons de dissimilation consonantique qui font la différence. Mais, paradoxalement, il y a moins de consonnes vélaires devant ces suffixes à voyelle postérieure que devant les suffixes en /i/ ou en /E/. On trouve, certes, quelques épenthèses ou interfixes en /k/ et en /g/ (HARGNIES (08) $\rightarrow$ Harnicot, MERY (77) $\rightarrow$ Mérycard, SABRES (40) $\rightarrow$ Sabringot, Boudes (63) $\rightarrow$ Boudigan), et quelques substitutions au profit de vélaires (CURTIL (71) $\rightarrow$ Curtigot) mais globalement le rapprochement entre consonne et voyelle s'est exercé ou bien en direction de -ois (dans la mesure où l'accroche consonantique commande le choix du suffixe), ou bien en direction des palatales (dans la mesure le choix du suffixe infléchit l'accroche consonantique). Il y a deux raisons, nous semble-t-il, à ce paradoxe. Les consonnes vélaires sont peu nombreuses en français et leur fréquence est assez peu élévée. Les consonnes latentes, en particulier, sont très rarement des vélaires et les suffixes évaluatifs n'en comportent pas. Or ce sont les premières qui inspirent les épenthèses et les seconds qui fournissent la plupart des interfixes. Les épenthèses et les interfixes en vélaire (cf. exemples ci-dessus et prusco, poiscaille, (ar)bicot, boursicoter...) sont d'autant plus remarquables et ne s'expliquent que par l'harmonisation entre consonne et voyelle. Mais la contrainte de série, qui privilégie l'attraction des sous-séries déjà bien fournies, ne joue pas en leur faveur et explique leur rareté. L'introduction d'une consonne palatale devant un suffixe à voyelle postérieure (-ot, -on, ard, -at...), d'autre part, est une solution moins satisfaisante du point de vue de l'harmonie entre consonne et voyelle, mais elle aboutit néanmoins à une postériorisation et elle satisfait l'harmonie (contrastive) entre voyelles puisque la palatale en question est généralement un yod qui, au contact du suffixe, joue le même rôle qu'un /i/ dans la syllabe précédente.

La même raison explique vraisemblablement le paradoxe symétrique qu'est la sous-représentation des palatales devant les suffixes à voyelle palatale -ais et -ain. Ce n'est pas le yod en tant que glide qui est malvenu devant ces suffixes (un mot comme pied n'est pas plus dysphonique que pré ou blé), ni le yod en tant que consonne palatale, c'est le /i/ qu'il y a en lui. Si devant un /E/ l'harmonie contrastive est mieux satisfaite par un /O/ ou un /A/ que par un /i/, la défaveur du /i/ entraîne celle du yod. La concurrence de 
-ien, qui présente une solution facile après yod grâce à l'haplologie, explique en partie la sousreprésentation de -ais pour ce type de bases, mais pas le fait que devant -ain et les autres suffixes en /E/ les accroches en palatale présentes dans le thème $B$ de la base soient deux fois sur trois éliminées du radical dans le dérivé (cf. Tableau 2).

\section{$5 \quad$ Elargissements}

Il faudrait évidemment vérifier si les contraintes que nous pensons avoir mises en évidence se manifestent en dehors du corpus examiné. Dans deux directions au moins : (i) les autres gentilés, ceux du corpus 2, plus hétérogènes ; (ii) les dérivés français en dehors des gentilés. Pour la première, disons d'un mot, faute de pouvoir détailler davantage, que les formations proprement françaises - forme francisée du toponyme, suffixe français - ne se distinguent pas des gentilés de l'hexagone. Dans les couples Algérois / Oranais, Pékinois / Cantonais, la répartition des deux variantes est la même que pour Lillois et Lyonnais. Pour des créations sans doute plus récentes, le /i/ de Syrte a appelé -ois (Syrtois), le /a/ de Misrata a appelé -ais (Misratais) :

Les gens de Syrte ont refusé que les Misratais entrent dans leur ville [...] la branche syrtoise d'Ansar Al-Charia [...] (Le Monde, 21.02.2016 : 12)

A propos des gentilés formés avec d'autres suffixes, on observera brièvement ceux en -Vte, qui ouvrent des perspectives au-delà du français. Et en dehors des gentilés, on proposera quelques éléments sur les dérivés formés avec -on, -ot, -aut et -eau.

\subsection{Les gentilés en -Vte}

Le suffixe grec -tês formait principalement, sur base verbale, nominale ou adjectivale, des noms d’humains fondés sur un rapport agentif, métonymique ou méronymique. Dont, déjà, des gentilés. Un processus de réanalyse engagé dès le grec ancien a agrégé progressivement au suffixe la voyelle ou le complexe voyelle-consonne qui le précédaient immédiatement, et qui appartenaient initialement au matériau thématique de la base. Il en est résulté, pour le français, d'un côté le suffixe -iste, promis à un brillant avenir, et son petit frère moins chanceux -aste (enthousiaste, cinéaste); de l'autre les suffixes -ite, -ète, -eute, -ate, -ote qui constituent ce que Malkiel (1970) appelle un " groupe (group) » dans lequel un même « pilier (pillar) » consonantique est précédé par une " gamme (gamut) » de voyelles. Quelle que soit la voyelle, les constructions dans lesquelles entrent ces suffixes ont les mêmes caractéristiques sémantiques et catégorielles. Dans le lexique français, ce sont des formations anciennes transmises par la voie savante (cénobite, proxénète, thérapeute, pirate, zélote) et des créations modernes forgées dans diverses langues, surtout comme gentilés et parfois dans d'autres domaines (jésuite, uniate, préraphaélite).

Les gentilés en -Vte constituent donc un ensemble hétérogène où se côtoient des formations du grec ancien, d'autres qui ont circulé principalement dans la lingua franca de la Méditerranée orientale où elles ont pu être formées initialement dans diverses langues, d’autres enfin, plus récentes, qui relèvent de la sphère anglo-saxonne globalisée. La voyelle y est le plus souvent /i/ ou / $\mathrm{j} /$, plus rarement /a/ (Eléate, Spartiate, Ravennate) ou /ع/ (Eginète, Magnète, Lisboète). Comme pour -ois / -ais, la répartition des formes est à la fois géographique et conditionnée phonologiquement. -ite s'est diffusé surtout au MoyenOrient, de la Palestine (Hiérosolymite) à la Mésopotamie (Ninivite), puis vers le nord (Moscovite), l'est (Annamite) et le sud (Yéménite). -ote autour de la mer Egée (Smyrniote, Piréote) et vers l'ouest (Corfiote, Sofiote, Benghaziote). D'un point de vue phonologique, -ite est présent surtout après /A/ et /0/ (Sybarite, Annamite, Sodomite, Moscovite...) tandis que -ate et -ote suivent surtout /i/ et /E/ (Urbinate, Ravennate, Epirote, Cairote) ou sont précédés d'un yod, présent dans la base (Asiate, Candiote) ou apporté par une épenthèse (Spartiate, Corfiote) ${ }^{17}$. Outre les dérivés où le /i/ du radical devient / $\mathrm{j} /$ dans le dérivé, on trouve dans notre corpus 22 dérivés dont le radical, à finale vocalique, est en hiatus devant la voyelle du suffixe. Après /a/ ou /o/, cette voyelle est toujours /i/ (Sabaïte, Méroïte) tandis que /e/ est toujours suivi de /a/ ou de /o/ (Eléate, Piréote). Le conditionnment phonologique joue non seulement pour le choix de la voyelle dans la gamme de suffixes, mais aussi pour le choix d'un de ces suffixes là où $a$ priori on ne l'attendait pas. En Afrique du Nord, où les gentilés en -Vte sont rares, une base en /i/ (Bougie, Safi) attire -ote (Bou- 
giote, Safiote), tandis que symétriquement des bases en /0/ susciteront une dérivation en -ite dans des régions où ces suffixes sont peu diffusés (Tokyoïte, Osloïte, Buffaloüte, Quitoüte ${ }^{18}$ ).

Les origines diverses de ces formations laissent supposer que la contrainte phonologique qui détermine les contrastes n’est pas particulière au français. La prédilection pour la séquence /io/ apparaît dès le grec ancien où ce sont des bases en -ia qui donnent des dérivés en -iôtês au lieu de -iatês ou -iêtês régulièrement attendus : patriôtes, stratiôtês, Italiôtês, Massaliôtês... Par la suite, la fréquence d'un /i/ ou d'un yod devant -ote a fini par faire percevoir -iote comme une variante du suffixe, voire pour sa forme standard. Mais la séquence symétrique /oi/, moins fréquente mais elle aussi privilégiée (cf. exemples cidessus) ne correspond pas à une variante et montre bien que le contraste fonctionne dans les deux sens et n'est pas particulier au français. Buffaloïte est anglo-américain et le curieux Quitoita apparaît sous la plume d'Abraham Cowley, poète anglais du $17^{\mathrm{e}}$ siècle ${ }^{19}$.

$$
\begin{aligned}
& \text { buffaloite [...] the real word for people from buffalo...not buffalonians like some peo- } \\
& \text { ple might purport definithing.com/buffaloite/ } \\
& \text { The Quitoita with this provision stor'd / Can pass the vast and cloudy Andes o'er } \\
& \text { https://books.google.fr/books?id=BW87AQAAIAAJ }
\end{aligned}
$$

\subsection{Autres effets de contraste dans la suffixation française}

Une étude antérieure (Roché, 2003) fondée sur un corpus de dérivés en -on avait observé dans le lexique général les mêmes effets d'harmonie constrastive que dans les gentilés formés avec ce suffixe. C'est une voyelle d'avant qui est privilégiée dans la dernière syllabe du radical, un /i/ surtout ou bien un yod dans l'accroche offerte au suffixe. D'où, par exemple, dans le type corbillon $\leftarrow$ CORBEILLE, la même substitution de voyelle que dans Vouvrillon $\leftarrow$ VOUVRAY commenté plus haut. Comme dans les gentilés, on observe un certain nombre de dérivés en -ion (croupion, gâtion, porion, tordion, trublion...), qu'on peut interpréter comme le résultat d'une épenthèse ou comme des variantes du suffixe, et qui s'ajoutent aux formations empruntées (ludion, lampion, vibrion...). La contrainte de taille privilégiant par ailleurs les radicaux dissyllabiques, un grand nombre de bases monosyllabiques sont allongées par un interfixe (-ill-, -ich-, -ign-) qui combine la voyelle souhaitée et une accroche en palatale ou prépalatale. (bottillon, carpillon, Cendrillon, cotillon, croisillon, grappillon, gravillon, moinillon, portillon, postillon, tardillon (ou tardichon), tatillon, taurillon... ; bonichon, drôlichon, folichon, cornichon, maigrichon, pâlichon, ratichon... ; fumignon, lumignon ${ }^{20}$, maquignon, salignon...). La voyelle de ces bases étant elle-même, majoritairement, un /A/ ou un /O/, le contraste porte aussi, le plus souvent, sur les deux premières syllabes. De sorte qu'un grand nombre de dérivés suivent une même courbe mélodique impliquant leurs trois syllabes, comme s'ils s'alignaient sur des lexèmes hérités ou d'origine obscure déjà dotés de cette caractéristique (papillon, carillon, potiron...) ${ }^{21}$. L’âne des Petites filles modèles ne s’appelle pas par hasard Cadichon.

Tout porte à croire que les mêmes observations pourraient être faites sur les suffixations en /0/ (-ot, aud, -eau). Pour s'en tenir aux accidents morphophonologiques qui s’opposent à la contrainte de fidélité, on y trouve, comme dans les gentilés, les mêmes épenthèses ou interfixes en yod ((ai)griotte, coffiot 'coffrefort', corniaud, crapouillot, fafiot, frio(t) 'froid', gaviot 'gosier', glaviot(er), godillot, loupiot, maigriot, normiot 'normalien', pégriot, pleuvioter, rabiot, raboliot, rafiot, salopiaud, sorbonniot...) ou en vélaire

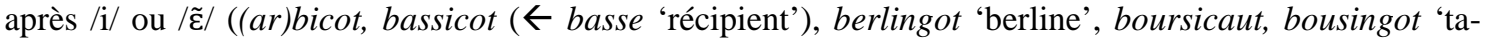
page', moricaud, palicot 'piège à poissons', saligaud...; fricot(er), boursicoter, fourchicoter, machicoter, ravigoter, tournicoter, emberlificoter...). Une étude plus approfondie ferait sans doute apparaître, également, une influence de la dernière voyelle du thème dans le choix du suffixe évaluatif : chariot face à charrette, petiot face à grandet, etc.

La manifestation la plus caractéristique de l'attirance mutuelle entre /i/ /j/ et /o/, aux antipodes des gentilés hellénisants en -iote d'un point de vue socio-linguistique bien qu'avec avec le même résultat, est la variante "régionale "-iau de -eau : fabliau, nobliau, flûtiau, bitoniau, les affûtiaux, les attifiaux, et la servante du châtiau, qui sont l'équivalent dans le lexique général du type Morvandiau rencontré plus haut ${ }^{22}$. On a dit « variante de -eau » parce qu'effectivement c'est une forme en -eau qu'on trouve souvent à côté dans la langue normée, mais beaucoup de ces dérivés en -iau sont également écrits -iot (massiau / massiot, roussiau / roussiot, sauteriau / sauteriot, etc.) et inversement on trouve rabiau et rafiau à côté 
des plus courants rabiot et rafiot. Il y a toute une circulation entre les différentes finales en /o/, qui, globalement, ont tendance à suivre un /i/ ou à se voir précéder d'un yod.

Ces formes en -iau sont moquées comme «paysannes » et l’on est frappé, parmi celles en -iot, -iaud, -icot, etc. relevées plus haut, par la proportion de termes populaires ou argotiques. Ce n'est sans doute pas une coïncidence : par rapport à langue normée, qui donne la priorité aux contraintes de fidélité, la langue populaire est davantage sensible aux contraintes phonologiques, qui s'y manifestent plus nettement. Elles se révèlent également dans des mots d'emprunt ou d'origine obscure qui ont subi de multiples transformations, ou dans des formations encore plus atypiques : artichaut, abricot, panicaut, calicot, haricot, asticot(er)..., billebaude, chiquenaude, gringuenaude..., artiflot, gourdiflot, à tire larigot... La plasticité dont ont fait preuve ces formations n'est pas aléatoire, elle leur a permis de s'approcher d'un certain idéal mélodique.

Il faudrait enfin observer symétriquement ce qui se passe devant les suffixes comportant une voyelle d'avant. Devant -et(te) (Plénat, 2005), il se pourrait que la diffusion de l'interfixe -oun- (blagounette, grippounette, larmounette, siestounette, tristounette...) ait la même origine. Elle est étrange a priori, puisque les contraintes lexicales favorisent -in- (putinette s'aligne sur cousinette) et que -ou(ne) est rare en tant que suffixe. Mais -oun- a l'avantage d'introduire un contraste arrière / avant (et arrondi / écarté) entre les voyelles. Avec -in(e), des indices laissent supposer que le schéma mélodique de Trissotin, Guillotin, Ripolin, Cidrolin et Bibi Fricotin, symétrique de celui de Cadichon, soit privilégié. A suivre.

\section{Conclusions et perspectives}

Dans la construction des gentilés, le choix du suffixe - pourquoi -ais plutôt que -ois ou -ois plutôt que -ais, pourquoi tel ou tel autre suffixe populaire - et la formation du radical - encore plus capricieuse, en apparence, que dans le reste du lexique - sont un défi pour qui voudrait les formuler en termes de règles. Nous pensons qu'ils sont le résultat du jeu des contraintes énumérées plus haut, qu’on ne pouvait évidemment décrire ici dans son ensemble. On n'a fait, pour deux ou trois d'entre elles, qu'observer les données et proposer des hypothèses susceptibles d'en rendre compte. Ces données confirment l'importance du conditionnement phonologique. Il suffit pour cela de voir les tableaux 1 et 2 : il n'y aurait pas autant de différences entre les suffixes, et pour un même suffixe entre environnements différents, si leur répartition ne dépendait que des autres paramètres. Elles confirment ce qu'on savait de la contrainte dissimilative dans son aspect le plus documenté et de celle qui harmonise l'attaque consonantique de la syllabe et le noyau vocalique. Elles nous ont conduit à formuler l'hypothèse d'une autre face - positive de la contrainte dissimilative, qui favorise entre les voyelles une harmonie fondée sur les contrastes haut/bas et avant/arrière. Le corpus sur lequel nous nous sommes appuyés est assez vaste, si nos analyses sont justes, pour rendre cette hypothèse plausible. Mais les recherches doivent être poursuivies, et la réflexion approfondie, pour qu'elle puisse être confirmée.

Plusieurs directions doivent pour cela être explorées. Dans les gentilés, il faut croiser les effets des contraintes phonologiques avec ceux des autres paramètres qui commandent le choix du suffixe et la forme du radical. Tout laisse à penser que l'essentiel se joue dans un va-et-vient entre ces contraintes et les contraintes lexicales - expansion en tache d'huile dans les sous-séries géographiques et par effet de rime dans les sous-séries caractérisées par une homophonie en amont du suffixe. Dans le lexique français, il faut rechercher les effets de contraste vocalique semblables à ceux que nous avons observés dans les gentilés. Les éléments dont on dispose déjà à propos des suffixations en -on et en /0/ donnent pour cela des indices prometteurs. Il faut enfin confronter les observations portant sur le français à celles qu'on peut faire sur d'autres langues. L’harmonie vocalique y est surtout connue sous la forme qui privilégie l’homogénéité, mais la recherche des contrastes doit aussi s’y trouver, comme le laissent supposer les dérivations en -Vte.

\section{Références bibliographiques}

Bonami, O., et Boyé, G. (2003). Supplétion et classes flexionnelles dans la conjugaison du français. Langages, 152, 102-126. 
Clements, N. (1993). Lieu d'articulation des consonnes et des voyelles : une théorie unifiée. In B. Laks et A. Rialland (dir.), Architecture des représentations phonologiques. Paris : CNRS Editions, 101-145.

Dauzat, A., et Rostaing, Ch. (1963). Dictionnaire étymologique des noms de lieux en France. Paris : Guénégaud.

Eggert, E. (2002). La dérivation toponymes-gentilés en français : mise en évidence des régularités utilisables dans le cadre d'un traitement automatique. Thèse de doctorat, Université François Rabelais (Tours) et Westfäliche Wilhelms-Universität (Münster).

Lignon, S., et Roché, M. (2011). Entre histoire et morphophonologie, quelle distribution pour -ien vs -éen ? In M. Roché, G. Boyé, N. Hathout, S. Lignon et M. Plénat, Des unités morphologiques au lexique. Paris / Londres : Hermès-Lavoisier, 191-250.

Malkiel, Y. (1970). Patterns of Derivational Affixation in the Cabraniego Dialect of East-Central Asturian. Berkeley: University of California Press.

Merlet, L. (1882). Dictionnaire des noms vulgaires des habitants de diverses localités de la France. Chartres : PétrotGarnier (réimp. Nîmes : Lacour, 1996).

Plénat, M. (2005). Rosinette, cousinette, putinette, starlinette, chipinette. Décalage, infixation et épenthèse devant -ette. In I. Choi-Jonin, M. Bras, A. Dagnac et M. Rouquier (éd.), Questions de classification en linguistique: méthodes et descriptions. Mélanges offerts au Professeur Christian Molinier. Berne : Peter Lang, 275-298.

Plénat, M. (2008). Quelques considérations sur la formation des gentilés. In B. Fradin (éd.), La raison morphologique. Hommage à la mémoire de Danièle Corbin. Amsterdam : John Benjamins, 155-174.

Plénat, M. (2015). Dissimilatory phenomena in French word-formation. In P. O. Müller, I. Ohnheiser, S. Olsen \& Fr. Rainer (eds.), Word-Formation. An International Handbook of the Languages of Europe. Berlin / New York: De Gruyter Mouton (coll. HSK), 944-955.

Roché, M. (2003). De la « bonne formation » des dérivés en -on. Cahiers de Grammaire, 28, 91-112.

Roché, M. (2009). Un ou deux suffixes ? Une ou deux suffixations ? In B. Fradin, F. Kerleroux et M. Plénat (dir.), Aperçus de morphologie du français. Saint-Denis : Presses Universitaires de Vincennes, 143-173.

Roché, M. (2010). Base, thème, radical. Recherches Linguistiques de Vincennes, 39, 95-133.

Roché, M. (2015). Interfixes in Romance. In P. O. Müller, I. Ohnheiser, S. Olsen \& F. Rainer (eds.), WordFormation. An International Handbook of the Languages of Europe. Berlin / New York: De Gruyter Mouton (coll. HSK), 551-568.

Roché, M., et Plénat, M. (2014). Le jeu des contraintes dans la sélection du thème présuffixal. In F. Neveu et al. (éd.), $4^{\mathrm{e}}$ Congrès Mondial de Linguistique Française. Paris : ILF, 1863-1878.

Rolland de Denus, A. (1889). Dictionnaire des appellations ethniques de la France et de ses colonies. Paris : Emile Lechevallier.

\footnotetext{
${ }^{1}$ On appellera indifféremment «suffixes » toutes les formes mobilisées, qu’il s’agisse véritablement de suffixes différents ou de variantes d'un même suffixe (sur cette question, voir Lignon et Roché (2011)).

${ }^{2}$ Ces formations ne doivent pas être confondues avec les gentilés qui sont de simples métonymies du toponyme (Barcelonnette pour BarCelonnette (04), par exemple). Dans La Guerre des boutons, de Louis Pergaud, les habitants (ou seulement les enfants?) de Velran sont appelés «les Velrans" et ceux de Longeverne «les Longerverne(s) ». On ne sait si ce procédé est particulier à ce roman ou s'il s’agit d’un usage plus général, mais nous n'avons pas trouvé de tels gentilés, dans les listes consultées, en dehors de quelques cas particuliers (dans l'exemple ci-dessus, il semble que les Barcelonnettes aient été surtout les émigrants originaires de l'Ubaye installés au Mexique et que le gentilé proprement dit soit plutôt Barcelonettais ou Barcelonettain, qui figurent également dans les listes). Les formations illustrées en (1b) et (6a) sont différentes puisqu'elles font jouer une alternance thématique pour former le féminin quand le toponyme a une forme masculine (Filain $\rightarrow$ Filaine) ou le masculin quand il a une forme féminine (VALLORCINE $\rightarrow$ Vallorcin), comme les formations nominales par inversion du genre (GuILlOTIN $\rightarrow$ guillotine) et les adjectifs de relation du type PIETON $\rightarrow$ (rue) piétonne. On pourrait les analyser comme des conversions, ou comme des suffixations avec haplologie, mais quelle que soit l'étiquette l'essentiel est que ce type de formation ne fonctionne que si le toponyme a une finale suffixoïde, plus précisément celle d'un suffixe pouvant devenir la marque d'un gentilé. Seule compte la forme de surface : si elle satisfait la condition requise pour figurer dans la série dérivationnelle souhaitée, une forme déjà présente dans la famille dérivationnelle, fût-elle celle de la base, peut faire l'affaire.

${ }^{3}$ La plupart des gentilés sont assez récents. Sauf coutumes locales, seules les provinces et les villes ou les bourgs de quelque importance devaient autrefois en être dotés. Les relevés de Merlet (1882) et de Rolland de Denus (1889) laissent supposer que c'était le cas à leur époque. Aujourd'hui encore, les listes restent lacunaires parce que certaines
} 
localités n'en ont pas (et pour d’autres on doute que le gentilé adopté par le conseil municipal soit réellement en usage). Quoi qu'il en soit, il est impossible de les dater puisque les dictionnaires étymologiques les ignorent.

${ }^{4}$ Un Alsacien consulté pour savoir comment on prononce les gentilés en -heimois (/عmwa/ ? /ajmwa/ ?), entre autres, nous a répondu : « On ne les emploie pas. On dit -heimer /ajmər/, même en contexte français ».

${ }^{5}$ Dans les exemples, les départements français sont identifiés par leur numéro, les régions françaises par un $\mathrm{F}$. $\mathrm{Bw}=$ Belgique wallonne, $\mathrm{Sr}=$ Suisse romande, $\mathrm{Q}=$ Québec. Quand le toponyme est une expression complexe (ClermontFerrand, Brive-la-Gaillarde), on n'a retenu que l'élément qui sert de base au dérivé (Clermont-ois, Briv-iste).

${ }^{6}$ Comme plusieurs suffixes font alterner une voyelle orale (au féminin) et une voyelle nasale (au masculin), on a regroupé sous $/ 0 /$ non seulement /o/ et $/ \mathrm{\jmath} /$ mais aussi $/ \tilde{\mathrm{J}} /$; sous $/ \mathrm{A} /: / \mathrm{a} /, / \mathrm{a} /$ et $/ \tilde{a} /$; sous $/ \mathrm{E} /: / \mathrm{e} /, / \varepsilon /$ et $/ \tilde{\varepsilon} /$ de -ain(e); sous /i/: : i/ et / $/ \tilde{\varepsilon} /$ de -in(e).

${ }^{7}$ Dans la base de données, on a considéré qu'aux finales en /i/ de la forme libre correspondaient régulièrement des thème $\mathrm{B}$ en $/ \mathrm{j} /$, comme dans MARIE $\rightarrow$ Marion, JOLI $\rightarrow$ joliesse (et en /ij/ après attaque branchante). Lorsqu'un /i/ suit /n/ (GlatignY) ou /j/ (Chantilly), que le yod résultant de la semi-vocalisation est absorbé par la consonne qui précède. De la même façon, on a postulé que les toponymes en -ay, -ey, -oy ont systématiquement un thème $\mathrm{B}$ en /عj/ ou en $/$ waj/.

${ }^{8}$ Etymologiquement, ReILly est l'équivalent du méridional ReilHAC : un nom d’homme suffixé en -acum, comme un grand nombre de toponymes en $-y$ (Dauzat-Rostaing).

${ }^{9}$ Paradoxalement, on trouve plusieurs exemples d'épenthèse en /z/ ( BEAulieu (07) $\rightarrow$ Beaulieusais, MonTAuliEU (26) $\rightarrow$ Montaulieusais, VILLEDIEU (86) $\rightarrow$ Villedieusois, LE RHEU (35) $\rightarrow$ Rheusois), alors que les sifflantes, on vient de le voir, sont indésirables dans cette position. Mais ces épenthèses - ce n'est pas un hasard - interviennent après une finale en $/ \varnothing /$. C'est la même épenthèse qu'on rencontre dans la formation de banlieusard, parce que l'alternance $/ \varnothing / \sim / \varnothing \mathrm{z} /$, à cause des adjectifs en -eux, -euse, est la plus fréquente pour cette voyelle. Pour ces gentilés, l'effet de série a été plus fort que la contrainte dissimilative.

${ }^{10}$ L'interprétation des interfixes en chwa est plus complexe. Comme ce chwa est généralement amuï, l'effet de l'interfixe s'exerce surtout sur les consonnes. S'il s'ajoute au radical, la voyelle qui précède le suffixe reste inchangée (VANNEs (56) $\rightarrow$ Vannetais). S'il se substitue à la dernière rime, c'est la voyelle de la syllabe préfinale qui devient celle qui précède le suffixe (AudEux (25) $\rightarrow$ Audelais).

${ }^{11}$ L'haplologie n'est pas obligatoire (cf. angiite, lobbyiste), mais les exemples de ce type sont très rares (aucun parmi les gentilés) et dans ce cas le premier /i/ reste voyelle. La séquence /ji/ n'est possible qu’à l'initiale (yin, yiddish) ou après voyelle (taillis).

${ }^{12}$ Si l'on adopte le schéma de Clements (1993), il serait plus juste de parler de voyelles dorsales (les voyelles d'arrière) et de voyelles radicales (les voyelles basses). Mais cela revient au même quant à l'opposition qui nous intéresse ici par rapport aux voyelles d'avant (coronales).

${ }^{13}$ Comme les gentilés sont souvent donnés au masculin pluriel (Les habitants du Morvan sont les Morvandiaux), on ne sait pas toujours quelle est la forme féminine. Face à Morvandeau, elle est théoriquement Morvandelle. Mais, à cause sans doute de la trop forte allomorphie entre le masculin et le féminin, l'alternance -eau / -elle n'est pas toujours respectée (cf. par exemple l'allomorphie «réduite»/o/ /od/ de Couzeau, -eaude $\leftarrow$ CouzE (24)). Face à Morvandiau, on trouve Morvandiaute, Morvandiaude (mais le masculin peut aussi être écrit Morvandiaud), Morvandiote (qui entraîne le masculin vers Morvandiot, lui aussi attesté).

${ }^{14}$ Quand ils sont donnés avec un féminin en -ouse, ce sont des variantes méridionales de -eux. Avec un féminin en -oune, des variantes (principalement méridionales) de -on. Quand on ne dispose que d'un masculin pluriel écrit -ous, on ne peut pas savoir duquel il s'agit.

${ }^{15}$ Les voyelles hautes d'avant, dans cette position, sont à $86 \%$ des /i/ et à $14 \%$ des /y/, dans notre corpus, et la question des glides ne se pose pratiquement que pour le yod. Seul /j/ peut se trouver en finale de la forme libre (Corbeil, Versailles), évidemment conservé au thème $\mathrm{B}$, tandis que des finales en $/ \mathrm{u} /$ et en $/ \mathrm{w} /$ sont inconnues du français. Quant aux glides par semi-vocalisation, ce sont essentiellement des yods puisque les finales en /i/, dans la forme libre, sont très fréquentes et donnent systématiquement un $/ \mathrm{j} /$ au thème $\mathrm{B}$ quand il n’y a pas de consonne latente tandis que les finales en $/ y /$ et en $/ \mathrm{u} /$ sont beaucoup plus rares et que leur semi-vocalisation est moins systématique.

${ }^{16}$ Les chiffres sont différents de ceux qu'on obtient en additionnant les lignes correspondantes des tableaux 1 et 2 puisqu'il faut soustraire les dérivés qui ont à la fois une accroche en palatale et une voyelle haute d'avant dans la syllabe précédente.

${ }^{17}$ Avec, évidemment, des interférences entre /i/ et yod suivant la langue considérée. Dans le cas de Cairote, c'est une diphtongue /ai/ qui devait se trouver là où le français a un $/ \varepsilon /$.

${ }^{18}$ Le gentilé « officiel » est plutôt Quiténien, inspiré de l'espagnol Quiteño, mais Quitoïte est bien attesté : 
A seulement $2 \mathrm{~h}$ de route de Quito, c'est incroyable de trouver un endroit aussi authentique ; c'est d'ailleurs un des lieux de villégiature de week-end pour de nombreux quitoïtes.

http://biketsworldtour.com/2015/02/07/mindo/

${ }^{19}$ On ne sait si ce Quitoita a été forgé par Cowley ou s'il s’agit d’un gentilé espagnol (ou néo-latin) qui aurait précédé (ou concurrencé) Quiteño.

${ }^{20}$ Les étymologies données par Wartburg ou Guiraud pour lumignon sont inutilement compliquées. Il s’agit plus vraisemblablement d'un dérivé construit sur le radical de lumière, allumer avec l'interfixe -ign- qu'on retrouve dans fumignon, maquignon, salignon ou dans le patronyme Trotignon. -ign- est une variante de -in- qui apparaît devant une voyelle basse d'arrière (cf. FIN $\rightarrow$ fignoler, troufignon, ou encore mignon, mignard sur le même phonesthème min- que minet).

${ }^{21}$ Cas particulier : les dérivés en -aillon (moussaillon, buvaillon, avocaillon...), où l’on peut voir un « suffixe composé » (Roché, 2009) puisque le segment -aill-, à la différence des éléments neutres que sont les interfixes, garde une valeur sémantique (péjorative) qui se combine avec la valeur diminutive de -on. Mais sur le plan morphophonologique on y retrouve la même courbe mélodique qui va de /a/ à / ̃̃ en passant par /j/.

${ }^{22}$ Un yod peut aussi être introduit devant /o/ dans le radical : dépiauter est construit sur peau. 